Elevation-Derived Watershed Basins and Characteristics for Major Rivers of the Conterminous United States

Scientific Investigations Report 2008-5153

U.S. Department of the Interior

U.S. Geological Survey 



\section{Elevation-Derived Watershed Basins and Characteristics for Major Rivers of the Conterminous United States}

By S.K. Poppenga and B.B. Worstell

Scientific Investigations Report 2008-5153 


\section{U.S. Department of the Interior DIRK KEMPTHORNE, Secretary}

\section{U.S. Geological Survey \\ Mark D. Myers, Director}

\section{U.S. Geological Survey, Reston, Virginia: 2008}

For product and ordering information:

World Wide Web: http://www.usgs.gov/pubprod

Telephone: 1-888-ASK-USGS

For more information on the USGS — the Federal source for science about the Earth, its natural and living resources, natural hazards, and the environment:

World Wide Web: http://www.usgs.gov

Telephone: 1-888-ASK-USGS

Any use of trade, product, or firm names is for descriptive purposes only and does not imply endorsement by the U.S. Government.

Although this report is in the public domain, permission must be secured from the individual copyright owners to reproduce any copyrighted materials contained within this report.

Suggested citation:

Poppenga, S.K., and Worstell, B.B., 2008, Elevation-derived watershed basins and characteristics for major rivers of the conterminous United States: U.S. Geological Survey Scientific Investigations Report 2008-5153, 27 p. 


\section{Contents}

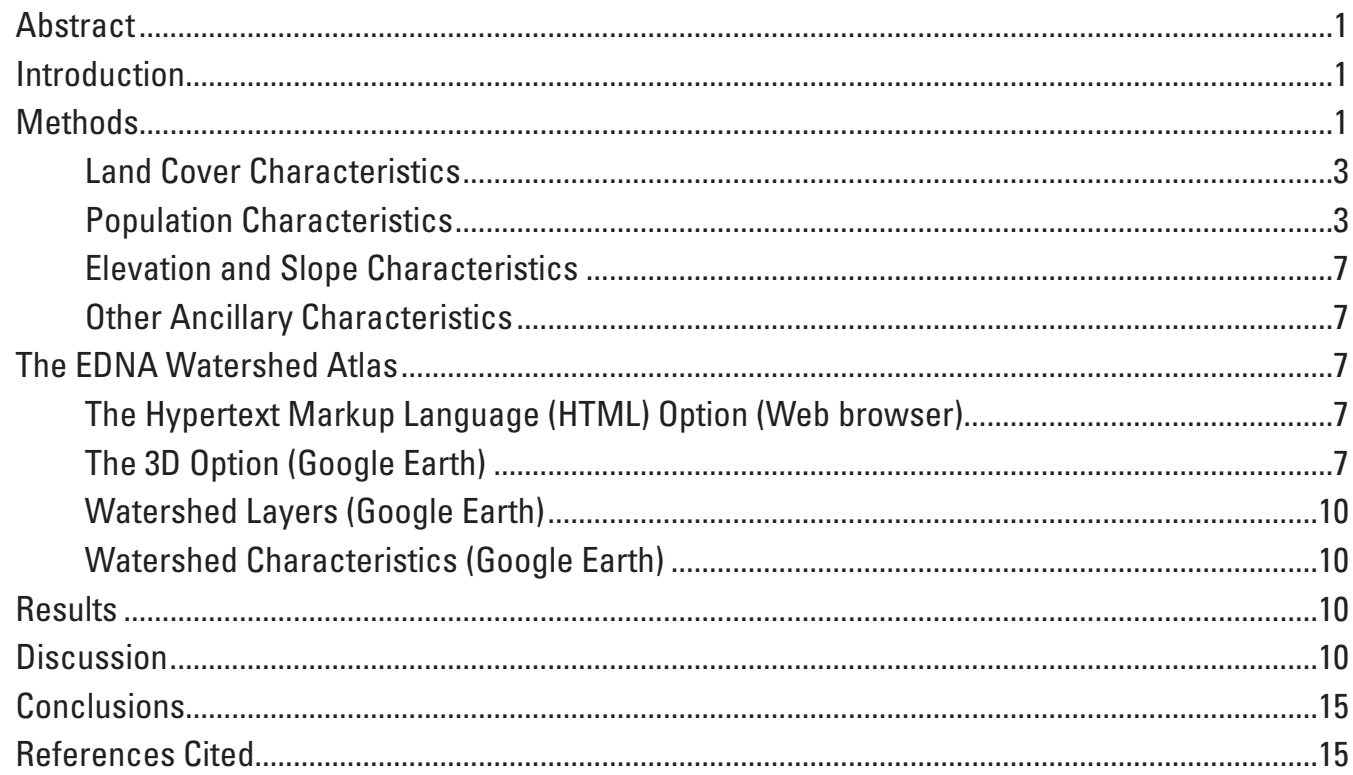

\section{Figures}

1. Web image showing geographic information system (GIS) layers for Elevation Derivatives for National Applications (EDNA) seamless database ..

2-3. Maps showing:

2. Elevation-derived watersheds for major rivers of the conterminous

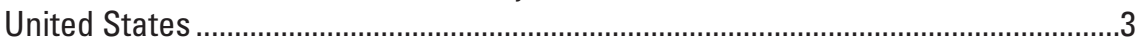

3. Reach catchments within an elevation-derived watershed .....................................4

4-9. Web images showing:

4. EDNA Watershed Atlas Web portal is accessible in a standard Web browser (HTML link) or with a three-dimension option, such as Google Earth (KML version link)

5. The EDNA Watershed Atlas is accessible from the EDNA Web site ......................17

6. Land cover characteristics for the Chesapeake Bay watershed............................18

7. Slope characteristics for the Chesapeake Bay watershed ....................................19

8. Population characteristics for the Chesapeake Bay watershed ............................20

9. Characteristics for the Chesapeake Bay watershed accessible in Google Earth

10-15. Maps showing:

10. Land cover characteristics for the Chesapeake Bay watershed, 1992..................22

11. Land cover characteristics for the Chesapeake Bay watershed, 2001 ...................23

12. Population characteristics for the Chesapeake Bay watershed, 1990 ..................24

13. Population characteristics for the Chesapeake Bay watershed, 2000 ..................25

14. Elevation characteristics for the Chesapeake Bay watershed ..............................26

15. Slope characteristics for the Chesapeake Bay watershed ....................................27 


\section{Tables}

1. Elevation-derived watersheds for major rivers of the conterminous United States........4

2. Land cover characteristics, in percent, for elevation-derived watersheds, 1992 ............5

3. National Land Cover Data classification schemes, 1992 ..................................................

4. Land cover characteristics, in percent, for elevation-derived watersheds, 2001 ............8

5. National Land Cover Data classification schemes, 2001 .................................................10

6. Population characteristics for elevation-derived watersheds, 1990 .................................11

7. Population characteristics for elevation-derived watersheds, 2000 .................................12

8. Elevation characteristics for elevation-derived watersheds........................................13

9. Slope characteristics, in degrees, for elevation-derived watersheds.............................14

10. National Inventory of Dams (NID) “Purposes” attribute categories. ................................15

\section{Conversion Factors}

\begin{tabular}{lcl}
\hline Multiply & By & To obtain \\
\hline & Length & \\
\hline centimeter $(\mathrm{cm})$ & 0.3937 & inch (in.) \\
meter $(\mathrm{m})$ & 3.281 & foot $(\mathrm{ft})$ \\
kilometer $(\mathrm{km})$ & 0.6214 & mile $(\mathrm{mi})$ \\
kilometer $(\mathrm{km})$ & 0.5400 & mile, nautical $(\mathrm{nmi})$ \\
meter $(\mathrm{m})$ & 1.094 & yard $(\mathrm{yd})$ \\
\hline & Area & \\
\hline square meter $\left(\mathrm{m}^{2}\right)$ & 0.0002471 & acre \\
square kilometer $\left(\mathrm{km}^{2}\right)$ & 247.1 & acre \\
square centimeter $\left(\mathrm{cm}^{2}\right)$ & 0.001076 & square foot $\left(\mathrm{ft}^{2}\right)$ \\
square meter $\left(\mathrm{m}^{2}\right)$ & 10.76 & square foot $\left(\mathrm{ft}^{2}\right)$ \\
square centimeter $\left(\mathrm{cm}^{2}\right)$ & 0.1550 & square inch $\left(\mathrm{ft}^{2}\right)$ \\
square kilometer $\left(\mathrm{km}^{2}\right)$ & 0.3861 & square mile $\left(\mathrm{mi}^{2}\right)$ \\
\hline & Flow rate & \\
\hline cubic meter per second $\left(\mathrm{m}^{3} / \mathrm{s}\right)$ & 70.07 & acre-foot per day $(\mathrm{acre}-\mathrm{ft} / \mathrm{d})$ \\
cubic meter per second $\left(\mathrm{m}^{3} / \mathrm{s}\right)$ & 35.31 & cubic foot per second $\left(\mathrm{ft}^{3} / \mathrm{s}\right)$ \\
cubic meter per second per & 91.49 & cubic foot per second per square \\
square kilometer $\left[\left(\mathrm{m}^{3} / \mathrm{s}\right) / \mathrm{km}^{2}\right]$ & 22.83 & million gallons per day $(\mathrm{Mgal} / \mathrm{d})$ \\
\hline cubic meter per second $\left(\mathrm{m}^{3} / \mathrm{s}\right)$ & &
\end{tabular}

Vertical coordinate information is referenced to the North American Vertical Datum of 1988 (NAVD 88).

Horizontal coordinate information is referenced to the North American Datum of 1983 (NAD 83). Elevation, as used in this report, refers to distance above the vertical datum. 


\title{
Elevation-Derived Watershed Basins and Characteristics for Major Rivers of the Conterminous United States
}

\author{
By S.K. Poppenga ${ }^{1}$ and B.B. Worstell ${ }^{2}$
}

\begin{abstract}
The U.S. Geological Survey Earth Resources Observation and Science Center Topographic Science Project has developed elevation-derived watershed basins and characteristics for major rivers of the conterminous United States. Watershed basins are delineated upstream from the mouth of major rivers by using the hydrologic connectivity of the Elevation Derivatives for National Applications (EDNA) seamless database. Watershed characteristics are quantified by integrating ancillary geospatial datasets, including land cover, population, slope, and topography, with elevation-derived watershed boundaries. The results are published in an online EDNA Watershed Atlas at http://edna.usgs.gov/watersheds. The atlas serves as a framework for evaluating and analyzing the physical, biological, and anthropogenic status of watersheds.
\end{abstract}

\section{Introduction}

In an effort to evaluate and analyze the health of the Nation's watersheds, the U.S. Geological Survey Earth Resources Observation and Science Center Topographic Science Project has developed elevation-derived watershed basins and characteristics for major rivers of the conterminous United States. Watershed basins are derived from hydrologically conditioned digital elevation models (DEMs) because the DEMs are precise, consistent, and unaffected by visual interpretations of cartographic data. Watershed characteristics are selected to provide metrics for land cover distributions, population density, topography, and impoundments for each watershed. This report describes the methods used to develop elevation-derived watershed basin delineations and characteristics and the watershed analysis capabilities within the Elevation Derivatives for National Applications (EDNA) Watershed Atlas (http://edna.usgs.gov/watersheds).

The hydrologic framework that facilitates analyses for the nationally integrated inventory of watershed basins and

\section{${ }^{1}$ U.S. Geological Survey.}

${ }^{2}$ SGT, contractor to the U.S. Geological Survey. Work performed under USGS contract 08HQCN0005. characteristics is the EDNA database (Franken and others, 2001). EDNA is a nationally consistent, 30 -meter resolution, multilayered database in which hydrologic flow representation and stream network connectivity are derived from the National Elevation Dataset (NED) (Gesch and others, 2002). The NED is a DEM of the land surface and is the basis for the EDNA database in that the DEM is a representation of the drainage surface of the United States. A geographic information system (GIS) is used to create hydrologic derivative layers by filling depressions in the DEM (from the NED), calculating flow direction and flow accumulation, and creating synthetic flow channels and reach catchments.

The EDNA is assembled as a seamless database with vertically consistent, hydrologic geospatial layers including hydrologically conditioned DEMs, flow direction, flow accumulation, synthetic flow channels, reach catchments, sinks, slope, aspect, and compound topographic index (CTI; fig. 1). Flow direction in EDNA channels is always from a higher elevation to a lower elevation, and the reach catchments always follow the drainage divide (Verdin and Greenlee, 2003). On the basis of this consistent information available on a national scale, watershed basins are delineated, and watershed characteristics are summarized for land cover, population, drainage area, minimum, maximum, and average elevation and slope for major rivers of the conterminous United States (fig. 2). The ability to access EDNA data layers as a seamless database facilitates the development of elevation-derived watersheds and characteristics.

\section{Methods}

An algorithm is written to delineate watersheds (contributing area) from any point in the United States by using EDNA's nationwide hydrologic connectivity. The underlying functionality of the watershed delineation is built on the EDNA reach catchments. During EDNA database development, reaches based on a 5,000-pixel drainage area are created from the DEM, and subsequently a catchment is produced for each one of the reaches (fig. 3). Watershed basins are delineated by selecting an outlet catchment (point) and automatically aggregating the upstream catchments (Kost 


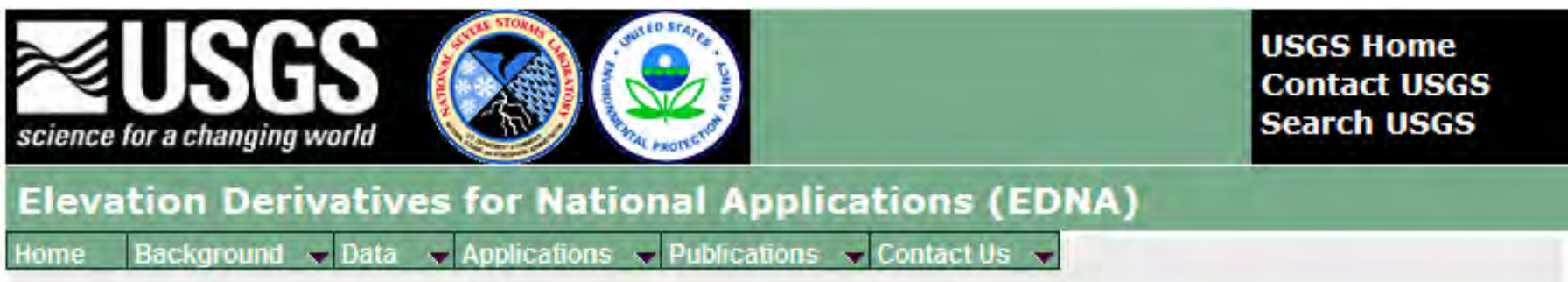

\section{EDNA Stage 1 Layers}

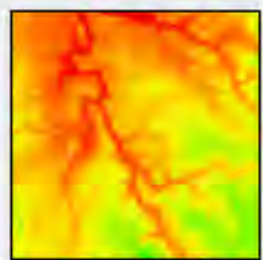

Filled DEM
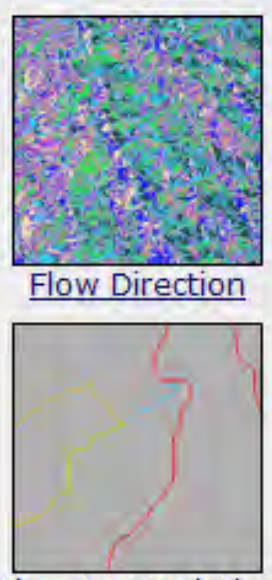

Flow Accumulation

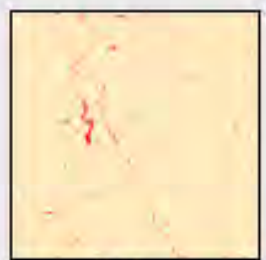

Sinks

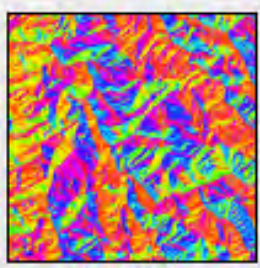

Aspect

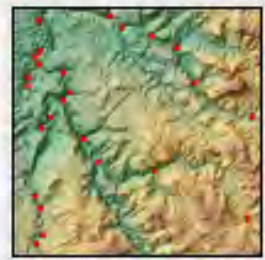

Reach Catchment Seedpoints
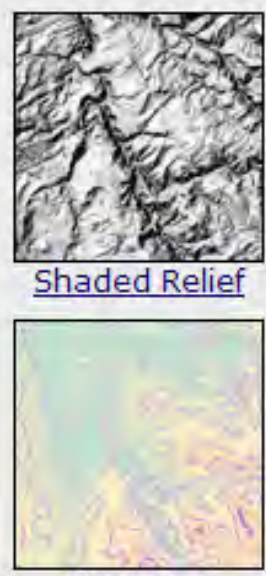

Contours

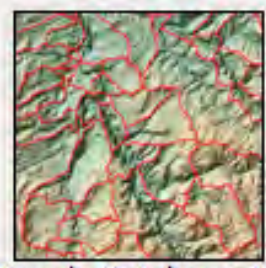

Reach Catchments

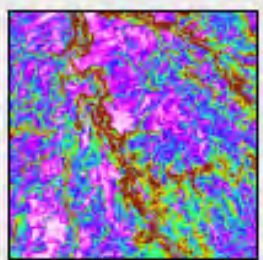

Slope

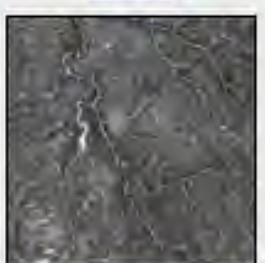

Compound Topographic Index (CTI)

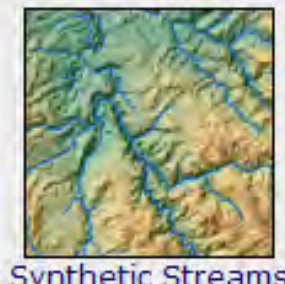

Figure 1. Geographic information system (GIS) layers for Elevation Derivatives for National Applications (EDNA) seamless database (available online at http://edna.usgs.gov/Edna/datalayers.asp).

and others, 2001). The output is a polygon that represents the contributing area of a watershed basin.

Elevation-derived watersheds are delineated by using the EDNA watershed tool, which is Web-enabled and contains integration capabilities for placing an outlet point for watershed delineation. The outlet location for each major river is interactively determined by using existing hydrography datasets with named rivers. The corresponding EDNA stream reach is used to seed an outlet point for the EDNA watershed tool, which processes the outlet point to create a delineation for the contributing area upstream from the point. Elevationderived watersheds are delineated for 77 major rivers of the United States from this tool (table 1 and fig. 2). The combined watersheds for these river systems cover most of the land surface of the conterminous United States. Each watershed delineation serves as a zone mask for computing and summarizing statistics for the watershed.
Watershed characterization includes methods that describe and define the physical, biological, and anthropogenic status of the watershed basin. The watershed characteristics summarized include land cover, population, elevation, and slope. Additional summaries include information about dams in the watersheds. To produce these characteristics, we used ESRI's ArcMap to convert watershed basins (polygons) to rasters (grids). Ancillary geospatial data are overlaid with each watershed grid to extract coincident land cover, population, drainage area, elevation, and slope grids. Watershed characteristics are summarized by quantifying the pixels in each contributing area by using the extracted coincident grids. The derived spatial and temporal characteristics describe the land cover (1992 and 2001), average population density (1990 and 2000, persons per square kilometer), drainage area (square kilometers), minimum and maximum elevation (meters), and minimum and maximum slope (degrees). The methods and 


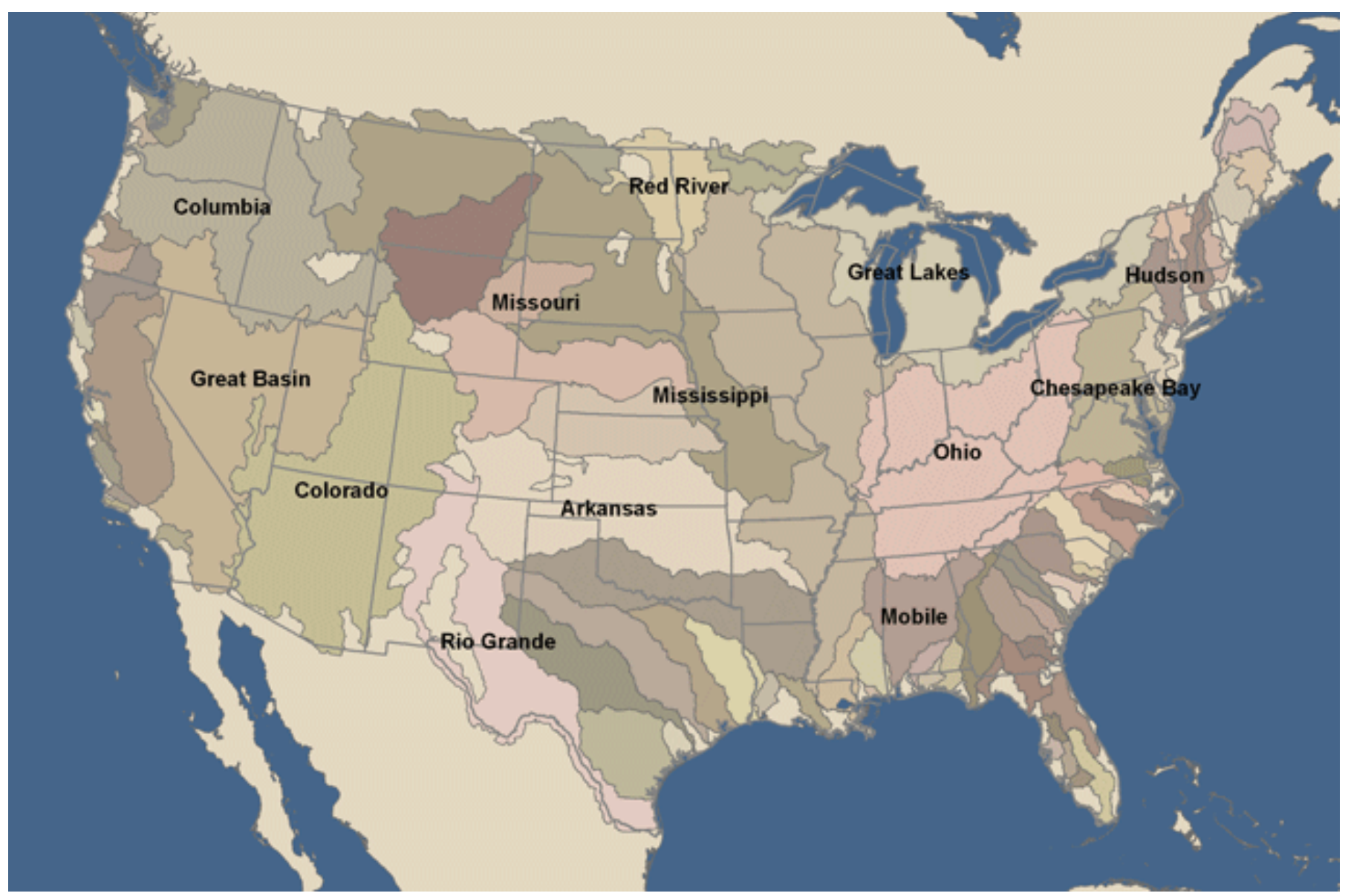

Figure 2. Elevation-derived watersheds for major rivers of the conterminous United States (available online at http://edna.usgs.gov/watersheds/html_index.htm).

examples for each of these characteristics are further described in the sections that follow.

\section{Land Cover Characteristics}

Distribution of land cover classes are computed for each watershed by using data from the 1992 and 2001 National Land Cover Data (NLCD; U.S. Geological Survey, 2007). These datasets are developed at 30-meter resolution. Each dataset was mapped with different methods and slightly different classes. The 1992 NLCD contains categories of land cover types, which are derived classifications of Landsat Thematic Mapper imagery from the mid-1990s; the 2001 NLCD contains classes, which are derived classifications of multiseason Landsat 5 and Landsat 7 imagery. The percentage of each NLCD category is computed and classified for each watershed on the basis of the Anderson Classification scheme. The 1992 and 2001 land cover characteristics are summarized in tables 2-5.

\section{Population Characteristics}

Population data from the Center for International Earth Science Information Network (CIESIN) are characterized for each watershed. The CIESIN is a nongovernmental organization at Columbia University, New York City. It develops datasets and analysis capabilities for studying a wide range of human and environmental interactions. CIESIN datasets include human population estimates for 1990 and 2000 called the Gridded Population of the World (GPWv3; Gridded Population of the World Version3, 2005). The dataset provides estimates of human population density in units of persons per square kilometer. 
Table 1. Elevation-derived watersheds for major rivers of the conterminous United States.

\begin{tabular}{lll}
\hline Altamaha & Hudson & Red River \\
Apalachicola & Kansas & Rio Grande \\
Arkansas & Kennebec & Roanoke \\
Atchafalaya & Klamath & Rogue \\
Biloxi & Lake Champlain & Sabine \\
Black & Merrimack & Sacramento/San Joaquin \\
Brazos & Mississippi & Saint John \\
Calcasieu & Missouri & Saint Johns \\
Caloosahatchee & Mobile & Salinas \\
Cape Fear & Navidad & San Francisco Bay \\
Chehalis & Neuse & San Gabriel \\
Chesapeake Bay & Nueces & Santa Maria \\
Cheyenne & Ochlockonee & Santa Ynez \\
Choctawhatchee & Ogeechee & Santee \\
Chowan & Ohio & Satilla \\
Colorado & Pajaro & Savannah \\
Colorado, Texas & Pamlico & Souris \\
Columbia & Pascagoula & Suwannee \\
Connecticut & Peace & Tampa Bay \\
Delaware & Pearl & Trinity \\
Edisto & Pee Dee & Umpqua \\
Eel & Penobscot & Waccamaw \\
Escambia & Platte & Withlacoochee \\
Everglades & Puget Sound & Yellow \\
Great Basin & Rainy & Yellowstone \\
Great Lakes & Red & \\
\hline & & \\
\hline
\end{tabular}

The GPWv3 dataset is developed globally using census data specific to each country. These country-specific data are converted to a uniform 2.5-arc-minute resolution raster dataset. Population density values for each census unit are developed for each country. These data are overlaid on a 2.5 -arc-minute grid to determine proportions of density values per grid cell (Deichmann and others, 2001).

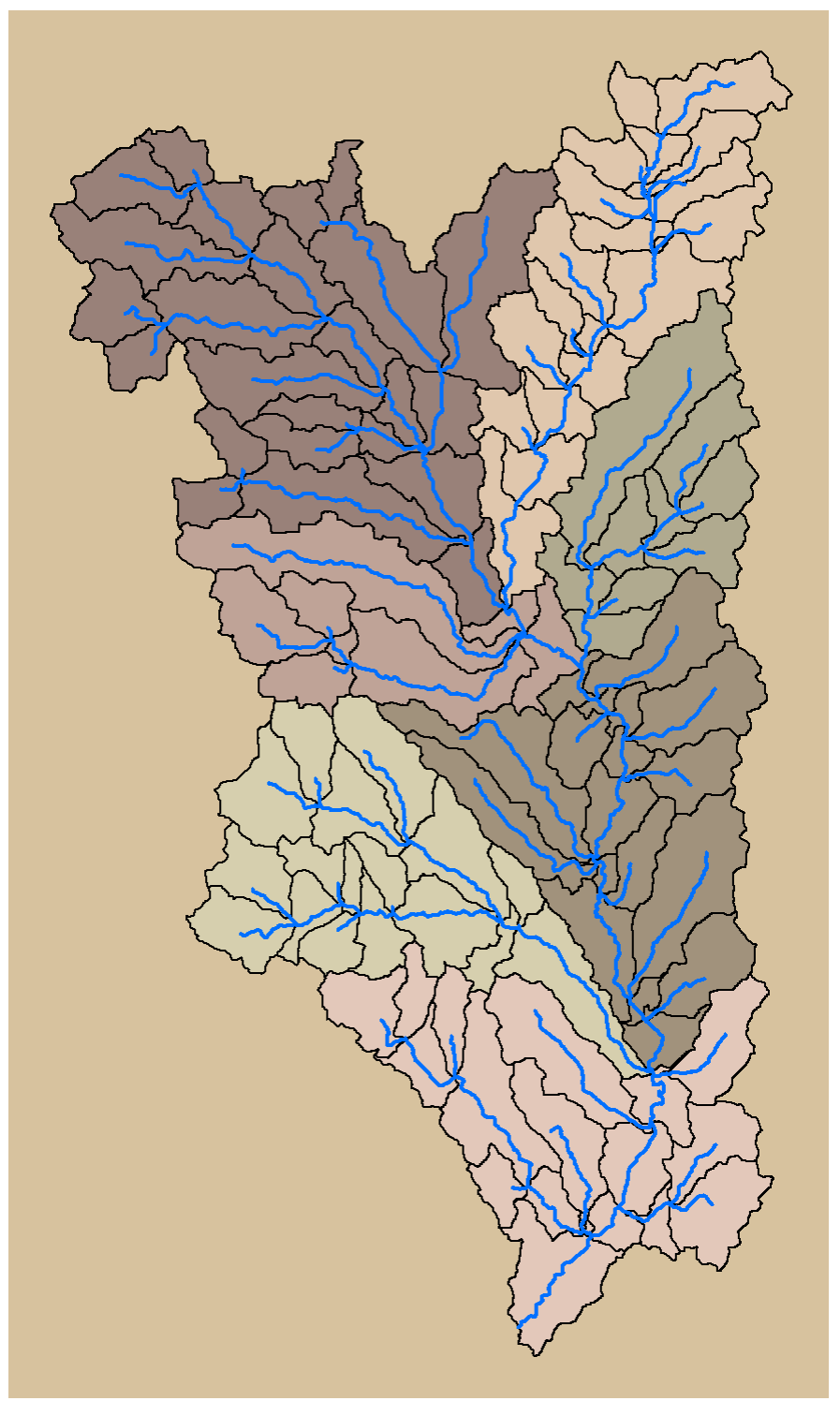

Figure 3. Reach catchments within an elevation-derived watershed.

GPWv3 population density datasets for 1990 and 2000 are used for estimating average population density for major U.S. watersheds. These data are projected into the USGS Albers Equal Area projection at a resolution of 3,845 meters by 3,845 meters. Population density samples (cells) for each watershed are summed and divided by the total number of cells in the watershed. The 1990 and 2000 population characteristics are summarized in table 6 and table 7, respectively. 
Table 2. Land cover characteristics, in percent, for elevation-derived watersheds, 1992 (National Land Cover Data (NLCD) from U.S. Geological Survey, 2007). NLCD 1992 classification schemes in table 3.

\begin{tabular}{|c|c|c|c|c|c|c|c|c|c|c|c|c|c|c|c|c|c|c|c|c|c|}
\hline Watersheds & 11 & 12 & 21 & 22 & 23 & 31 & 32 & 33 & 41 & 42 & 43 & 51 & 61 & 71 & 81 & 82 & 83 & 84 & 85 & 91 & 92 \\
\hline Altamaha & 1.2 & 0 & 1.7 & 0.4 & 0.8 & 0.1 & 0.3 & 5.4 & 21.9 & 24.8 & 10.8 & 0 & 0 & 0 & 8.1 & 15.5 & 0 & 0 & 0.5 & 8.4 & 0.2 \\
\hline Apalachicola & 2.0 & 0 & 1.6 & .4 & .9 & 0 & .1 & 4.2 & 21.8 & 17.5 & 17.6 & 0 & 0 & .1 & 8.6 & 15.5 & 0 & 0 & .4 & 9.1 & .4 \\
\hline Arkansas & 1.2 & 0 & .6 & .2 & .4 & .3 & 0 & .1 & 8.7 & 4.4 & 1.6 & 5.6 & 0 & 44.6 & 11.2 & 6.9 & 13.0 & .2 & .1 & .5 & .3 \\
\hline Atchafalaya & 6.2 & 0 & .9 & .1 & .7 & 0 & 0 & .2 & 3.5 & 3.4 & 1.4 & 0 & 0 & .4 & 10.4 & 20.5 & 2.9 & 0 & .7 & 19.9 & 28.9 \\
\hline Biloxi & 3.1 & 0 & 1.6 & .5 & 1.1 & 0 & 0 & 3.3 & 2.2 & 40.9 & 14.3 & 0 & 0 & 0 & 8.4 & 2.6 & 0 & 0 & 1.7 & 18.8 & 1.5 \\
\hline Black & .4 & 0 & 1.2 & .3 & .7 & 0 & .1 & 2.9 & 10.6 & 23.7 & 8.8 & 0 & 0 & 0 & 3.1 & 28.8 & 0 & 0 & .3 & 18.9 & .3 \\
\hline Brazos & 1.6 & 0 & .5 & .2 & .5 & .5 & .1 & 0 & 7.2 & 4.5 & .4 & 14.5 & 0 & 26.3 & 15.5 & 21.7 & 5.5 & .1 & .1 & .3 & .4 \\
\hline Calcasieu & 5.7 & 0 & 1.4 & .3 & 1.0 & .2 & .1 & 4.2 & 7.6 & 28.1 & 11.3 & 0 & 0 & .4 & 9.3 & 4.2 & 8.1 & 0 & .2 & 11.3 & 6.7 \\
\hline Caloosahatchee & 3.0 & 0 & 8.9 & 1.9 & 1.1 & .1 & .1 & 1.0 & 0 & 9.5 & 0 & 2.1 & 6.0 & 14.9 & 13.6 & 10.5 & 0 & 0 & .6 & 15.4 & 11.4 \\
\hline Cape Fear & 1.5 & 0 & 2.6 & .8 & 1.3 & .2 & .1 & 1.1 & 20.4 & 24.9 & 9.6 & 0 & 0 & 0 & 7.3 & 15.8 & 0 & 0 & .3 & 13.9 & .4 \\
\hline Chehalis & 1.6 & 0 & .9 & 0 & .6 & .1 & 0 & 6.6 & 18.9 & 45.1 & 16.0 & 1.0 & .2 & .5 & 6.7 & .6 & .1 & 0 & 0 & 1.0 & .2 \\
\hline Chesapeake Bay & 7.6 & 0 & 2.4 & .2 & 1.1 & 0 & .4 & .6 & 40.7 & 5.6 & 11.7 & 0 & 0 & 0 & 20.7 & 6.2 & 0 & 0 & .2 & 1.7 & .9 \\
\hline Cheyenne & .5 & 0 & .1 & 0 & .1 & .9 & .1 & 0 & .5 & 10.2 & 0 & 12.9 & 0 & 65.8 & 2.7 & .5 & 2.9 & 1.4 & .1 & .5 & .9 \\
\hline Choctawhatchee & 2.9 & 0 & .5 & .1 & .5 & 0 & 0 & 4.7 & 13.5 & 21.8 & 20.9 & 0 & 0 & 0 & 8.6 & 16.2 & 0 & 0 & .4 & 9.4 & .4 \\
\hline Chowan & 1.4 & 0 & .9 & 0 & .6 & 0 & 0 & 2.3 & 25.4 & 15.8 & 15.1 & 0 & 0 & 0 & 9.9 & 16.6 & 0 & 0 & .1 & 11.5 & .4 \\
\hline Colorado & .4 & 0 & .3 & 0 & .2 & 3.8 & .1 & 0 & 2.9 & 19.4 & .6 & 58.9 & 0 & 11.0 & 1.1 & .7 & .2 & 0 & 0 & .1 & .1 \\
\hline Colorado-Texas & .7 & 0 & .5 & .2 & .5 & .7 & 0 & 0 & 3.1 & 7.8 & 0 & 42.0 & 0 & 25.9 & 3.5 & 13.1 & 1.7 & 0 & .1 & 0 & .1 \\
\hline Columbia & 1.3 & .1 & .4 & 0 & .4 & 1.6 & 0 & 1.4 & 1.3 & 37.4 & 1.2 & 30.3 & .3 & 10.5 & 4.5 & 1.5 & 4.8 & 2.7 & 0 & .2 & .2 \\
\hline Connecticut & 2.3 & 0 & 3.2 & .5 & 1.7 & .2 & .1 & .6 & 38.0 & 14.7 & 24.0 & .2 & 0 & 0 & 2.3 & 6.4 & 0 & 0 & .9 & 3.5 & 1.3 \\
\hline Delaware & 8.2 & 0 & 5.6 & 1.2 & 1.9 & 0 & .5 & .2 & 35.4 & 4.1 & 14.0 & 0 & 0 & 0 & 16.7 & 6.7 & 0 & 0 & .6 & 2.8 & 2.0 \\
\hline Edisto & 2.9 & 0 & .7 & .3 & .3 & .1 & .1 & 4.5 & 10.8 & 26.5 & 9.3 & 0 & 0 & 0 & 2.6 & 23.0 & 0 & 0 & .1 & 16.8 & 1.9 \\
\hline Eel & .4 & 0 & .1 & 0 & .1 & .7 & 0 & .3 & 5.8 & 50.2 & 10.0 & 12.1 & 0 & 19.2 & 1.0 & 0 & 0 & 0 & 0 & 0 & 0 \\
\hline Escambia & .4 & 0 & .3 & 0 & .2 & 0 & .1 & 4.3 & 11.2 & 35.9 & 25.2 & 0 & 0 & 0 & 6.9 & 8.4 & 0 & 0 & .2 & 6.6 & .3 \\
\hline The Everglades & 13.7 & 0 & 3.1 & .7 & .9 & .1 & 0 & .7 & 0 & 4.3 & 0 & .3 & 3.3 & 13.6 & 5.7 & 12.9 & 0 & 0 & .2 & 15.7 & 24.6 \\
\hline Great Basin & 2.0 & 0 & .2 & 0 & .2 & 9.3 & .1 & 0 & .9 & 10.2 & .3 & 67.5 & 0 & 5.7 & 2.3 & .4 & .5 & 0 & 0 & 0 & .4 \\
\hline Great Lakes & 31.8 & 0 & 1.3 & .6 & .7 & 0 & .1 & .2 & 19.9 & 3.8 & 4.6 & 0 & 0 & .9 & 9.6 & 17.2 & 0 & 0 & .4 & 7.9 & 1.1 \\
\hline Hudson & 3.2 & 0 & 3.5 & 1.1 & 1.3 & 0 & .1 & 0 & 40.8 & 10.4 & 19.4 & 0 & 0 & 0 & 13.2 & 3.8 & 0 & 0 & .7 & 2.3 & .3 \\
\hline Kansas & 0.7 & 0 & 0.2 & 0.1 & 0.3 & 0.1 & 0 & 0 & 1.9 & 0.1 & 0.1 & 0.6 & 0 & 42.8 & 8.1 & 25.5 & 16.7 & 2.1 & 0.1 & 0.1 & 0.4 \\
\hline Kennebec & 6.0 & 0 & .7 & .1 & .6 & .1 & 0 & 2.1 & 30.3 & 22.3 & 27.4 & .3 & 0 & 0 & 1.9 & 4.4 & 0 & 0 & .3 & 2.5 & 1.1 \\
\hline Klamath & 1.5 & 0 & .1 & 0 & .2 & .7 & 0 & .6 & 1.2 & 62.2 & 3.1 & 14.1 & 0 & 8.3 & 2.8 & .5 & 2.5 & 0 & 0 & .1 & 2.1 \\
\hline Lake Champlain & 7.7 & 0 & 1.0 & .3 & 1.0 & .1 & .1 & .3 & 38.3 & 13.1 & 16.4 & .1 & 0 & 0 & 6.6 & 11.8 & 0 & 0 & .2 & 2.1 & 1.1 \\
\hline Merrimack & 4.9 & 0 & 6.2 & .9 & 3.0 & .1 & .1 & .4 & 30.8 & 17.3 & 23.6 & .1 & 0 & 0 & 1.6 & 5.0 & 0 & 0 & 1.1 & 3.1 & 1.6 \\
\hline Mississippi & 1.48 & 0 & .76 & .16 & .46 & .2 & .08 & .34 & 16.88 & 6.10 & 4.04 & 4.78 & 0 & 25.72 & 13.0 & 15.3 & 6.98 & 1.22 & .2 & 1.70 & .54 \\
\hline Missouri & 1.1 & 0 & .3 & .1 & .3 & .3 & 0 & .3 & 3.2 & 5.6 & .2 & 8.5 & 0 & 42.3 & 9.0 & 15.1 & 8.2 & 4.1 & .1 & .4 & 1.0 \\
\hline Mobile & 1.6 & 0 & .8 & .2 & .5 & 0 & .2 & 2.3 & 25.8 & 19.3 & 24.3 & 0 & 0 & 0 & 10.3 & 7.7 & 0 & 0 & .3 & 6.4 & .3 \\
\hline Navidad & 7.5 & 0 & .4 & .2 & .4 & .3 & .1 & .1 & 13.5 & 7.4 & .3 & 11.0 & 0 & 14.0 & 21.8 & 17.7 & .5 & 0 & .1 & .3 & 4.5 \\
\hline Neuse & 3.3 & 0 & 2.8 & .6 & 1.3 & .1 & .1 & .9 & 16.1 & 19.3 & 7.0 & 0 & 0 & 0 & 6.2 & 23.6 & 0 & 0 & .4 & 17.8 & .5 \\
\hline Nueces & 3.3 & 0 & .6 & .5 & .6 & .5 & .1 & 0 & 6.9 & 12.5 & .3 & 38.5 & 0 & 13.2 & 11.4 & 8.7 & 1.1 & .1 & .2 & .2 & 1.3 \\
\hline Ochlockonee & 1.6 & 0 & 1.6 & .2 & .4 & 0 & .1 & 8.9 & 2.6 & 23.7 & 7.3 & .3 & .3 & 1.9 & 3.2 & 19.2 & 0 & 0 & .1 & 26.9 & 1.6 \\
\hline Ogeechee & 1.3 & 0 & .8 & .3 & .5 & .1 & 0 & 5.6 & 10.8 & 26.9 & 7.4 & 0 & 0 & 0 & 3.0 & 26.3 & 0 & 0 & .2 & 16.2 & .8 \\
\hline Ohio & 1.4 & 0 & 1.6 & .2 & .7 & 0 & .3 & .3 & 43.4 & 4.2 & 7.6 & 0 & 0 & .1 & 19.2 & 19.4 & 0 & 0 & .5 & 1.0 & .1 \\
\hline Pajaro & .1 & 0 & 1.5 & .2 & .6 & 1.6 & 0 & 0 & 3.7 & 12.7 & 7.5 & 16.6 & 3.7 & 40.3 & 7.4 & 3.8 & 0 & .1 & .2 & 0 & 0 \\
\hline Pamlico & 1.5 & 0 & 1.7 & .2 & .7 & .1 & .1 & 1.4 & 21.9 & 20.2 & 8.3 & 0 & 0 & 0 & 7.9 & 21.0 & 0 & 0 & .3 & 14.6 & .3 \\
\hline Pascagoula & 1.1 & 0 & .5 & .1 & .4 & 0 & 0 & 3.6 & 11.1 & 32.6 & 22.7 & 0 & 0 & 0 & 11.4 & 5.4 & 0 & 0 & .2 & 10.2 & .6 \\
\hline Peace & 4.1 & 0 & 5.7 & 1.4 & 1.4 & .5 & 1.2 & .6 & 0 & 6.8 & 0 & 1.1 & 10.6 & 26.9 & 9.8 & 6.4 & 0 & 0 & .3 & 15.0 & 8.2 \\
\hline
\end{tabular}


Table 2. Land cover characteristics, in percent, for elevation-derived watersheds, 1992 (National Land Cover Data (NLCD) from U.S. Geological Survey, 2007). NLCD 1992 classification schemes in table 3. - Continued

\begin{tabular}{|c|c|c|c|c|c|c|c|c|c|c|c|c|c|c|c|c|c|c|c|c|c|}
\hline Watersheds & 11 & 12 & 21 & 22 & 23 & 31 & 32 & 33 & 41 & 42 & 43 & 51 & 61 & 71 & 81 & 82 & 83 & 84 & 85 & 91 & 92 \\
\hline Pearl & 16.3 & 0 & 1.9 & .5 & .7 & .1 & 0 & .9 & 11.8 & 21.3 & 12.3 & 0 & 0 & .1 & 14.1 & 4.7 & 0 & 0 & .4 & 6.8 & 8.0 \\
\hline Pee Dee & .9 & 0 & 2.3 & .4 & 1.1 & .1 & .1 & 1.2 & 23.7 & 17.3 & 10.8 & 0 & 0 & 0 & 10.2 & 20.7 & 0 & 0 & .3 & 10.8 & .1 \\
\hline Penobscot & 5.7 & 0 & .3 & .1 & .4 & .1 & 0 & 2.4 & 21.3 & 27.8 & 34.2 & .3 & 0 & 0 & .4 & 1.8 & 0 & 0 & .2 & 4.4 & .8 \\
\hline Platte & .9 & .1 & .6 & .1 & .4 & .2 & 0 & .1 & 1.7 & 6.2 & 0 & 11.8 & 0 & 50.0 & 4.6 & 14.5 & 3.6 & 3.0 & .1 & .2 & 1.8 \\
\hline Puget Sound & 16.3 & .9 & 3.6 & 0 & 1.2 & 2.5 & 0 & 4.1 & 8.7 & 45.1 & 7.9 & 2.6 & .2 & 2.4 & 3.4 & .3 & .2 & 0 & .2 & .3 & 0 \\
\hline Rainy & 9.6 & 0 & .1 & 0 & .3 & 0 & .2 & 1.1 & 17.0 & 10.8 & 12.4 & 1.8 & 0 & 0 & 2.4 & 1.7 & .4 & 0 & 0 & 38.7 & 3.4 \\
\hline Red & 2.1 & 0 & .6 & .1 & .4 & .2 & 0 & .7 & 14.0 & 11.3 & 7.9 & 5.2 & 0 & 16.8 & 12.3 & 15.2 & 7.8 & .1 & .1 & 4.8 & .4 \\
\hline Red River & 3.1 & 0 & .2 & .1 & .4 & 0 & 0 & .1 & 6.7 & .2 & .1 & .2 & 0 & 3.4 & 9.4 & 57.1 & 8.3 & 0 & .1 & 4.9 & 5.9 \\
\hline Rio Grande & .7 & 0 & .2 & .1 & .3 & 1.7 & 0 & 0 & .5 & 9.8 & .1 & 48.8 & 0 & 34.1 & 1.2 & 1.9 & .1 & 0 & 0 & 0 & .3 \\
\hline Roanoke & 2.3 & 0 & 1.8 & .1 & .7 & 0 & .1 & 1.3 & 39.1 & 14.2 & 12.7 & 0 & 0 & 0 & 16.3 & 6.0 & 0 & 0 & .1 & 5.1 & .2 \\
\hline Rogue & .4 & 0 & .5 & 0 & .3 & .2 & 0 & .8 & 2.1 & 69.1 & 11.5 & 2.9 & 0 & 6.4 & 5.0 & .2 & .4 & 0 & .1 & 0 & .2 \\
\hline Sabine & 4.6 & 0 & .9 & .3 & 1.0 & .1 & .1 & 2.3 & 17.6 & 15.8 & 21.9 & 0 & 0 & .5 & 20.9 & 2.1 & 1.8 & 0 & .1 & 6.5 & 3.4 \\
\hline $\begin{array}{l}\text { Sacramento/San } \\
\text { Joaquin }\end{array}$ & 1.6 & 0 & 1.6 & 0 & 0.6 & 2.2 & 0 & 0.1 & 3.0 & 29.3 & 4.0 & 13.1 & 6.0 & 20.7 & 6.4 & 6.3 & 4.3 & 0 & 0.1 & 0.1 & 0.6 \\
\hline Saint John & 3.3 & 0 & .3 & 0 & .3 & 0 & 0 & 3.3 & 23.8 & 26.2 & 33.2 & .5 & 0 & 0 & .3 & 5.7 & 0 & 0 & .2 & 2.4 & .5 \\
\hline Saint Johns & 7.6 & 0 & 6.8 & 2.0 & 1.7 & .2 & .1 & 3.7 & 0 & 29.9 & 0 & 1.3 & 3.1 & 9.7 & 2.7 & 7.6 & 0 & 0 & .3 & 15.7 & 7.4 \\
\hline Salinas & .2 & 0 & .6 & .1 & .4 & 3.4 & 0 & 0 & 1.3 & 12.4 & 3.7 & 16.0 & .8 & 47.9 & 4.9 & 7.1 & .9 & .1 & .3 & 0 & 0 \\
\hline San Francisco & 12.3 & 0 & 18. & .4 & 4.9 & 1.0 & .1 & 0 & 4.7 & 10.4 & 8.5 & 7.5 & .4 & 26.5 & 1.4 & .6 & .3 & 0 & 1.5 & 0 & .5 \\
\hline San Gabriel & .6 & 0 & 14.4 & 2.9 & 5.0 & 1.7 & 0 & 0 & .8 & 13.6 & 3.2 & 38.4 & .9 & 9.5 & 1.4 & 5.0 & .9 & .1 & 1.3 & .1 & .1 \\
\hline Santa Maria & 0 & 0 & .6 & .2 & .3 & 5.0 & 0 & 0 & 2.1 & 14.7 & 3.8 & 28.3 & 1.0 & 36.3 & 3.9 & 3.1 & .5 & .1 & .1 & 0 & 0 \\
\hline Santa Ynez & .4 & 0 & .7 & .2 & .3 & 1.3 & .3 & 0 & 1.3 & 15.4 & 8.3 & 35.0 & .6 & 29.2 & 2.9 & 3.3 & .4 & 0 & .3 & 0 & 0 \\
\hline Santee & 2.8 & 0 & 3.5 & .9 & 1.6 & .2 & .1 & 2.1 & 26.9 & 24.6 & 14.2 & 0 & 0 & 0 & 7.8 & 10.3 & 0 & 0 & .6 & 4.1 & .2 \\
\hline Satilla & 1.2 & 0 & .4 & .1 & .3 & 0 & 0 & 7.6 & 4.3 & 37.0 & 5.5 & 0 & 0 & 0 & 4.0 & 21.2 & 0 & 0 & .1 & 16.0 & 2.3 \\
\hline Savannah & 3.1 & 0 & 1.4 & .3 & .7 & .1 & .2 & 4.7 & 21.4 & 29.7 & 13.1 & 0 & 0 & 0 & 7.2 & 10.5 & 0 & 0 & .4 & 6.5 & .7 \\
\hline Souris & 1.3 & 0 & .2 & .1 & .4 & .4 & .1 & 0 & 3.2 & 0 & 0 & 1.2 & 0 & 26.9 & 2.0 & 10.4 & 42.4 & 7.4 & .1 & .1 & 3.9 \\
\hline Suwannee & .9 & 0 & 1.1 & .2 & .4 & .1 & .2 & 6.7 & 2.2 & 32.8 & 2.6 & .5 & .1 & 4.9 & 4.7 & 20.3 & 0 & 0 & .1 & 19.9 & 2.5 \\
\hline Tampa Bay & 14.6 & 0 & 12.3 & 6.2 & 4.0 & .4 & 1.1 & .9 & 0 & 6.5 & 0 & .6 & 5.7 & 15.8 & 4.2 & 6.6 & 0 & 0 & 1.1 & 15.1 & 4.9 \\
\hline Trinity & 5.4 & 0 & 3.8 & 2.0 & 2.3 & .2 & .1 & .5 & 12.4 & 5.5 & 9.8 & 1.4 & 0 & 8.7 & 35.3 & 5.6 & 2.6 & 0 & .7 & 2.0 & 1.7 \\
\hline Umpqua & .6 & 0 & .2 & 0 & .3 & .2 & 0 & 1.4 & 3.3 & 71.0 & 11.4 & 2.0 & 0 & 4.9 & 4.5 & 0 & .1 & 0 & 0 & 0 & .1 \\
\hline Waccamaw & 1.4 & 0 & .9 & .2 & .7 & .1 & .1 & 1.7 & 4.1 & 32.9 & 5.8 & 0 & 0 & 0 & 3.7 & 17.0 & 0 & 0 & .5 & 30.7 & .2 \\
\hline Withlacoochee & 2.2 & 0 & 7.7 & .8 & .9 & .2 & .2 & 3.0 & .3 & 21.9 & 0 & 2.3 & 2.2 & 16.5 & 2.5 & 15.0 & 0 & 0 & .2 & 17.9 & 6.1 \\
\hline Yellow & 2.0 & 0 & .4 & .1 & .7 & 0 & 0 & 6.2 & 10.5 & 34.3 & 18.7 & 0 & 0 & 0 & 6.6 & 10.4 & 0 & 0 & .4 & 9.2 & .4 \\
\hline Yellowstone & .6 & .1 & .1 & 0 & .1 & .5 & .1 & 1.1 & .8 & 12.8 & .1 & 27.0 & 0 & 46.9 & 1.9 & .6 & 3.2 & 3.4 & 0 & .3 & .4 \\
\hline
\end{tabular}


Table 3. National Land Cover Data classification schemes, 1992 (from U.S. Geological Survey, 2007).

\begin{tabular}{|ll}
\hline & \\
\hline 11 & Open Water \\
\hline 12 & Perennial Ice / Snow \\
\hline 21 & Low Intensity Residential \\
\hline 22 & High Intensity Residential \\
\hline 23 & Commercial / Industrial / Transportation \\
\hline 31 & Bare Rock / Sand / Clay \\
\hline 32 & Quarries / Strip Mines / Gravel Pits \\
\hline 33 & Transitional \\
\hline 41 & Deciduous Forest \\
\hline 42 & Evergreen Forest \\
\hline 43 & Mixed Forest \\
\hline 51 & Shrubland \\
\hline 61 & Orchards / Vineyards / Other \\
\hline 71 & Grasslands / Herbaceous \\
\hline 81 & Pasture / Hay \\
\hline 82 & Row Crops \\
\hline 83 & Small Grains \\
\hline 84 & Fallow \\
\hline 95 & Urban / Recreational Grasses \\
\hline 92 & Woody Wetlands \\
\hline & Emergent Herbaceous Wetlands \\
\hline
\end{tabular}

\section{Elevation and Slope Characteristics}

Elevation statistics are computed by using the EDNA filled elevation layer, a hydrologically conditioned DEM that contains no depressions. Zonal statistics are derived for each watershed drainage area and include minimum, maximum, and average elevation. Statistics for EDNA slope are computed in the same manner. The elevation and slope characteristics are summarized in table 8 and table 9 , respectively. Watershed basin characteristics derived from EDNA are among the most time-consuming to generate because of the size of the nationwide elevation and slope grids, each exceeding 55 gigabytes.

\section{Other Ancillary Characteristics}

The National Inventory of Dams (NID) database contains an inventory of more than 79,000 dams in the United States. The database is maintained by the U.S. Army Corps of Engineers and used for inspection, safety, and other applications by Federal and State agencies. A dam is included in the
NID database if it is a potential hazard or exceeds a height of 25 feet with 15 acre-feet of storage, or exceeds a height of 6 feet with 50 acre-feet of storage. Because dams can be used for multiple purposes, they are categorized as such in the NID database with a "Purposes" attribute (table 10). For each "Purpose" category assigned to each dam in a watershed, the percentage of dams used for each category is computed. Because some dams have multiple purposes, the percentages for the number of dams in a watershed do not always equal 100 percent. For example, a watershed with 100 dams might have 100 percent used for recreation, 60 percent used for flood control, and 1 percent used for hydroelectric generation.

\section{The EDNA Watershed Atlas}

The elevation-derived watershed basins and characteristics for major rivers of the conterminous United States are published in an online EDNA Watershed Atlas. The atlas serves as a framework for geographic analyses of watershed basins and characteristics and is a valuable tool for evaluating change within watersheds. The atlas is published in a standard Web browser and in a three-dimensional (3D) environment, such as Google Earth. The EDNA Watershed Atlas portal is at http://edna.usgs.gov/watersheds/ (fig. 4) and is accessible through the EDNA Web site at http://edna.usgs.gov (fig. 5).

\section{The Hypertext Markup Language (HTML) Option (Web browser)}

By selecting the HTML link on the EDNA Watershed Atlas portal (fig. 4), the elevation-derived watershed basins and characteristics can be accessed exclusively in a standard Web browser. Each watershed name is a Web link to that watershed's characteristics. For example, selection of the Chesapeake Bay watershed (link) redirects to the watershed characteristics for the Chesapeake Bay (fig. 6). Additional Chesapeake Bay watershed characteristics, such as land cover, population, elevation, slope, or dams can be accessed by selecting one of the EDNA watershed characteristics links (left side in fig. 6). Selection of the Slope link redirects to the Chesapeake Bay watershed slope characteristics (fig. 7). Other watersheds can be accessed by selecting the Return to Watershed Index link to return to the EDNA Watershed Atlas portal.

\section{The 3D Option (Google Earth)}

The EDNA Watershed Atlas can be accessed in 3D by selecting the $K M L$ link from the Watershed Atlas portal at http://edna.usgs.gov/watersheds/ (fig. 4). The 3D atlas portal looks similar to the Web portal; however, selection of the 3D links will result in a Keyhole Markup Language (KML) file loaded into Google Earth. There are two options in Google Earth: Watershed Layers and Watershed Characteristics. 
Table 4. Land cover characteristics, in percent, for elevation-derived watersheds, 2001 (National Land Cover Data (NLCD) from U.S. Geological Survey, 2007). NLCD 2001 classification schemes in table 5.

\begin{tabular}{|c|c|c|c|c|c|c|c|c|c|c|c|c|c|c|c|c|}
\hline Watersheds & 11 & 12 & 21 & 22 & 23 & 24 & 31 & 41 & 42 & 43 & 52 & 71 & 81 & 82 & 90 & 95 \\
\hline Altamaha & 1.2 & 0 & 5.9 & 2.6 & 0.7 & 0.3 & 0.7 & 20.0 & 24.8 & 5.1 & 0.9 & 9.7 & 10.6 & 6.4 & 10.5 & 0.7 \\
\hline Apalachicola & 1.9 & 0 & 5.5 & 2.6 & .7 & .4 & .3 & 19.7 & 24.0 & 3.9 & 4.0 & 5.5 & 9.0 & 12.3 & 9.6 & .4 \\
\hline Arkansas & 1.2 & 0 & 3.2 & .9 & .3 & .1 & .3 & 8.9 & 5.4 & .6 & 7.1 & 41.8 & 8.6 & 20.7 & .8 & .2 \\
\hline Atchafalaya & 5.5 & 0 & .5 & 2.9 & .4 & .2 & .2 & 0 & 2.3 & .6 & 1.3 & 1.1 & 6.8 & 23.8 & 45.8 & 8.6 \\
\hline Biloxi & 3.1 & 0 & 7.7 & 4.2 & 1.3 & .4 & .4 & 0 & 37.9 & 1.2 & 13.2 & 2.8 & 4.6 & .3 & 21.0 & 1.8 \\
\hline Black & .4 & 0 & 4.8 & 1.2 & .3 & .1 & .1 & 3.8 & 20.6 & 1.5 & 5.0 & 8.4 & 8.6 & 16.2 & 28.5 & .5 \\
\hline Brazos & 1.0 & 0 & 3.4 & .8 & .3 & .1 & .2 & 5.4 & 4.9 & .7 & 19.8 & 30.0 & 8.9 & 22.2 & 2.0 & .3 \\
\hline Calcasieu & 5.2 & 0 & 2.3 & 4.5 & .5 & .3 & .2 & .1 & 26.7 & 2.8 & 14.2 & 6.6 & 7.8 & 6.1 & 16.5 & 6.1 \\
\hline Caloosahatchee & 2.1 & 0 & 8.2 & 5.8 & 1.1 & .3 & .1 & 0 & 3.4 & 0 & 12.8 & 1.3 & 17.5 & 16.9 & 21.6 & 8.8 \\
\hline Cape Fear & 1.4 & 0 & 6.0 & 2.9 & .9 & .3 & .3 & 15.6 & 19.0 & 4.1 & 3.7 & 9.1 & 9.2 & 12.8 & 13.9 & .5 \\
\hline Chehalis & 1.4 & 0 & 4.6 & 1.6 & .5 & .2 & 2.3 & 4.1 & 51.1 & 6.0 & 11.2 & 5.2 & 4.8 & .9 & 4.4 & 1.7 \\
\hline Chesapeake Bay & 7.3 & 0 & 4.5 & 2.3 & .9 & .3 & .6 & 42.2 & 6.5 & 5.4 & .6 & .3 & 17.7 & 8.8 & 1.6 & .9 \\
\hline Cheyenne & .6 & 0 & .7 & .2 & .1 & 0 & 1.3 & .3 & 11.5 & .1 & 16.3 & 62.8 & 1.0 & 3.5 & 1.0 & .5 \\
\hline Choctawhatchee & 2.9 & 0 & 5.0 & 1.0 & .3 & .1 & .2 & 8.1 & 29.6 & 5.9 & 13.8 & 1.6 & 8.6 & 11.0 & 11.4 & .5 \\
\hline Chowan & 1.1 & 0 & 2.0 & .5 & .1 & 0 & 1.6 & 25.1 & 26.5 & 3.2 & .7 & 1.6 & 11.0 & 16.1 & 9.9 & .6 \\
\hline Colorado & .5 & .1 & .7 & .5 & .2 & 0 & 3.3 & 3.4 & 19.8 & .4 & 61.1 & 7.1 & 1.3 & .9 & .5 & .2 \\
\hline Colorado-Texas & .5 & 0 & 2.1 & .5 & .2 & .1 & .2 & 2.8 & 5.2 & .3 & 57.5 & 15.6 & 2.5 & 11.9 & .5 & .1 \\
\hline Columbia & 1.2 & 0 & 1.4 & .7 & .3 & .1 & .9 & .5 & 36.5 & .5 & 35.8 & 8.9 & 2.1 & 10.2 & .5 & .5 \\
\hline Connecticut & 2.0 & 0 & 4.5 & 2.7 & 1.4 & .3 & .2 & 37.2 & 17.7 & 21.2 & 1.4 & .2 & 5.1 & 2.1 & 3.6 & .3 \\
\hline Delaware & 7.9 & 0 & 6.8 & 4.4 & 2.2 & 1.0 & .9 & 38.5 & 3.5 & 4.4 & .2 & .1 & 11.7 & 12.5 & 4.0 & 1.9 \\
\hline Edisto & 2.6 & 0 & 4.1 & 1.1 & .2 & .1 & .1 & 8.1 & 25.4 & 2.6 & 2.9 & 11.7 & 7.9 & 11.6 & 19.9 & 1.8 \\
\hline Eel & .5 & 0 & 3.1 & .1 & .1 & 0 & .4 & 5.2 & 48.8 & 7.1 & 24.4 & 9.6 & .6 & 0 & .1 & 0 \\
\hline Escambia & .7 & 0 & 3.9 & .6 & .1 & 0 & .2 & 14.7 & 32.5 & 10.0 & 11.3 & 1.0 & 8.8 & 6.6 & 9.3 & .3 \\
\hline The Everglades & 12.0 & 0 & 4.1 & 1.8 & .6 & .2 & .1 & 0 & 2.0 & 0 & 3.9 & .9 & 11.7 & 15.8 & 18.7 & 28.2 \\
\hline Great Basin & 1.8 & 0 & .7 & .5 & .2 & 0 & 6.9 & 1.6 & 10.9 & .2 & 69.5 & 4.1 & 1.8 & 1.1 & .2 & .4 \\
\hline Great Lakes & 34.0 & 0 & 3.5 & 2.3 & .8 & .3 & .3 & 17.8 & 4.4 & 2.4 & 1.1 & 2.1 & 6.0 & 15.1 & 8.7 & 1.1 \\
\hline Hudson & 3.1 & 0 & 5.5 & 2.1 & 1.0 & .4 & .1 & 39.2 & 10.0 & 9.9 & 2.0 & .6 & 10.8 & 5.6 & 9.2 & .4 \\
\hline Kansas & .7 & 0 & 3.5 & .7 & .2 & .1 & .1 & 2.4 & 0 & 0 & 0 & 39.1 & 3.4 & 49.1 & .7 & .1 \\
\hline Kennebec & 5.6 & 0 & 2.3 & 1.1 & .4 & .1 & .5 & 23.9 & 20.3 & 28.2 & 6.3 & .8 & 2.5 & 1.3 & 5.8 & .9 \\
\hline Klamath & 1.7 & 0 & 1.7 & .3 & 0 & 0 & .9 & .8 & 55.2 & 1.8 & 21.4 & 8.9 & 2.3 & 3.4 & 0 & 1.5 \\
\hline Lake Champlain & 7.4 & 0 & 3.1 & 1.4 & .6 & .1 & .1 & 33.1 & 15.8 & 14.8 & 1.7 & .6 & 10.4 & 5.1 & 5.1 & .5 \\
\hline Merrimack & 4.4 & 0 & 5.4 & 5.0 & 3.6 & 0.8 & 0.4 & 28.4 & 19.2 & 20.1 & 1.3 & 0.3 & 4.6 & 1.0 & 4.8 & 0.9 \\
\hline Mississippi & 1.6 & 0 & 3.5 & 1.3 & .4 & .1 & .3 & 15.6 & 5.2 & 1.1 & 6.8 & 24.9 & 9.5 & 26.7 & 2.1 & .8 \\
\hline Missouri & 1.2 & 0 & 2.3 & .6 & .2 & .1 & .5 & 3.2 & 5.9 & .1 & 10.6 & 41.5 & 6.8 & 25.1 & 1.0 & .9 \\
\hline Mobile & 1.9 & 0 & 4.9 & 1.4 & .4 & .2 & .3 & 26.8 & 19.9 & 9.5 & 7.3 & 2.3 & 12.2 & 4.4 & 7.9 & .4 \\
\hline Navidad & 6.9 & 0 & 4.4 & .5 & .2 & .1 & .2 & 7.6 & 4.7 & .5 & 9.4 & 2.6 & 33.3 & 22.8 & 4.7 & 1.9 \\
\hline Neuse & 3.4 & 0 & 7.6 & 2.7 & 1.0 & .3 & .1 & 12.9 & 14.7 & 4.0 & 1.8 & 7.9 & 7.3 & 21.6 & 13.9 & .7 \\
\hline Nueces & 2.7 & 0 & 3.3 & 1.5 & .5 & .2 & .5 & 4.0 & 6.8 & .2 & 45.3 & 9.0 & 13.9 & 8.8 & 2.0 & 1.2 \\
\hline Ochlockonee & 1.0 & 0 & 4.6 & 1.1 & .2 & .1 & .2 & 8.0 & 26.1 & 7.8 & .3 & 6.1 & 3.9 & 13.6 & 25.6 & 1.5 \\
\hline Ogeechee & .6 & 0 & 4.7 & 1.5 & .4 & .1 & .3 & 11.8 & 24.3 & 4.6 & 1.4 & 11.2 & 5.9 & 15.0 & 16.3 & 1.9 \\
\hline Ohio & 1.5 & 0 & 5.8 & 2.1 & .7 & .3 & .2 & 46.2 & 2.5 & 2.1 & .9 & 2.3 & 15.3 & 19.3 & .6 & .1 \\
\hline Pajaro & .2 & 0 & 6.2 & 2.0 & 1.5 & .3 & .2 & 0 & 7.0 & 14.1 & 24.9 & 34.1 & 1.1 & 8.1 & .3 & .3 \\
\hline Pamlico & 1.5 & 0 & 5.6 & 1.4 & .5 & .2 & .1 & 17.9 & 18.8 & 4.4 & 2.4 & 7.9 & 9.2 & 20.1 & 9.8 & .4 \\
\hline Pascagoula & 1.3 & 0 & 4.4 & .9 & .3 & .1 & .1 & 6.8 & 30.2 & 14.6 & 13.1 & 1.0 & 10.3 & 1.9 & 14.5 & .6 \\
\hline Peace & 3.6 & 0 & 7.2 & 2.7 & .9 & .3 & 1.5 & 0 & 2.0 & 0 & 2.6 & 4.9 & 26.5 & 17.2 & 25.3 & 5.4 \\
\hline
\end{tabular}


Table 4. Land cover characteristics, in percent, for elevation-derived watersheds, 2001 (National Land Cover Data (NLCD) from U.S. Geological Survey, 2007). NLCD 2001 classification schemes in table 5. - Continued

\begin{tabular}{|c|c|c|c|c|c|c|c|c|c|c|c|c|c|c|c|c|}
\hline Watersheds & 11 & 12 & 21 & 22 & 23 & 24 & 31 & 41 & 42 & 43 & 52 & 71 & 81 & 82 & 90 & 95 \\
\hline Pearl & 16.2 & 0 & 4.3 & 2.2 & .7 & .3 & .2 & 5.2 & 17.3 & 6.3 & 10.0 & 1.1 & 10.9 & 2.5 & 17.4 & 5.4 \\
\hline Pee Dee & .8 & 0 & 6.4 & 2.0 & .5 & .2 & .1 & 22.7 & 14.5 & 2.7 & 2.2 & 8.2 & 14.4 & 11.9 & 13.1 & .2 \\
\hline Penobscot & 4.6 & 0 & 1.4 & .6 & .2 & .1 & .5 & 16.1 & 22.5 & 33.1 & 7.0 & .7 & .9 & 1.1 & 9.9 & 1.4 \\
\hline Platte & .8 & .1 & 2.2 & .8 & .3 & .1 & .4 & .9 & 7.0 & .1 & 15.5 & 46.8 & 1.3 & 21.3 & 1.2 & 1.3 \\
\hline Puget Sound & 15.0 & .9 & 4.1 & 4.2 & 1.6 & .7 & 3.2 & 2.6 & 43.8 & 6.6 & 7.6 & 3.1 & 2.9 & .6 & 1.7 & 1.2 \\
\hline Rainy & 9.1 & 0 & .8 & .2 & 0 & 0 & .2 & 31.4 & 21.2 & .3 & 3.2 & 1.0 & 1.4 & 1.2 & 21.5 & 8.6 \\
\hline Red & 2.0 & 0 & 3.3 & 1.4 & .2 & .1 & .2 & 10.4 & 14.7 & 3.6 & 14.0 & 19.6 & 7.4 & 16.5 & 6.3 & .2 \\
\hline Red River & 4.1 & 0 & 3.9 & .5 & .1 & 0 & 0 & 7.7 & .9 & 0 & .2 & 4.5 & 8.3 & 59.9 & 2.8 & 6.9 \\
\hline Rio Grande & .5 & 0 & 1.0 & .6 & .2 & .1 & 1.0 & .8 & 10.0 & .1 & 63.7 & 18.2 & 1.1 & 2.0 & .4 & .3 \\
\hline Roanoke & 2.0 & 0 & 4.6 & 1.7 & .4 & .2 & .3 & 41.8 & 13.0 & 3.9 & 2.0 & 3.5 & 18.4 & 3.9 & 4.1 & .1 \\
\hline Rogue & .3 & 0 & 2.7 & .9 & .3 & .1 & .5 & .2 & 65.5 & 2.6 & 18.6 & 3.6 & 3.0 & .9 & .5 & .1 \\
\hline Sabine & 4.1 & 0 & 3.7 & 3.1 & .5 & .2 & .1 & 5.9 & 21.7 & 6.6 & 8.4 & 5.7 & 15.6 & 2.2 & 18.9 & 3.1 \\
\hline $\begin{array}{l}\text { Sacramento/San } \\
\text { Joaquin }\end{array}$ & 1.5 & 0 & 2.9 & 1.2 & 1.1 & .2 & 2.6 & 1.4 & 27.1 & 1.5 & 17.8 & 20.5 & 2.8 & 18.0 & .2 & 1.0 \\
\hline Saint John & 2.8 & 0 & .8 & .4 & .1 & 0 & .5 & 15.9 & 20.9 & 33.0 & 10.1 & .8 & 1.0 & 4.7 & 8.4 & 6 \\
\hline Saint Johns & 6.7 & 0 & 9.0 & 4.2 & 1.3 & .4 & .2 & .7 & 24.1 & .7 & 2.2 & 8.3 & 10.3 & 4.8 & 22.0 & 5.1 \\
\hline Salinas & .3 & 0 & 5.0 & .7 & .5 & .1 & 2.4 & 0 & 5.2 & 8.8 & 29.4 & 37.4 & 1.3 & 7.9 & .6 & .3 \\
\hline San Francisco & 12.0 & 0 & 8.1 & 7.7 & 14.0 & 4.2 & 0 & 0 & 4.6 & 17.7 & 11.5 & 18.2 & 0.2 & 0.6 & 0.2 & 1.0 \\
\hline San Gabriel & .6 & 0 & 11.6 & 11.9 & 13.6 & 2.1 & .6 & 0 & 10.0 & 1.6 & 31.1 & 12.2 & 1.5 & 2.7 & .3 & 0 \\
\hline Santa Maria & 0 & 0 & 2.6 & .8 & .4 & 0 & 2.6 & 0 & 8.7 & 8.4 & 51.2 & 17.3 & 1.7 & 5.6 & .3 & .4 \\
\hline Santa Ynez & .5 & 0 & 4.1 & 1.0 & .6 & 0 & .3 & 0 & 15.8 & 13.5 & 41.4 & 15.7 & 1.8 & 4.1 & .4 & .7 \\
\hline Santee & 2.9 & 0 & 7.8 & 3.5 & 1.0 & .5 & 6 & 31.3 & 19.8 & 2.0 & 1.3 & 6.9 & 15.4 & 1.5 & 5.4 & .2 \\
\hline Satilla & .8 & 0 & 4.3 & 1.5 & .3 & .1 & .1 & 3.6 & 30.5 & 2.7 & 1.5 & 11.3 & 4.9 & 14.3 & 21.0 & 3.0 \\
\hline Savannah & 3.1 & 0 & 5.3 & 1.9 & .5 & .2 & .9 & 25.6 & 25.5 & 2.6 & 1.2 & 10.1 & 12.0 & 2.9 & 7.6 & .8 \\
\hline Souris & 3.2 & 0 & 4.2 & .5 & .1 & 0 & .1 & 2.3 & 0 & 0 & 0 & 20.9 & 8.4 & 54.1 & .5 & 5.7 \\
\hline Suwannee & .6 & 0 & 4.6 & 1.1 & .2 & .1 & .2 & 4.0 & 26.8 & 5.0 & .8 & 11.7 & 7.8 & 13.3 & 22.3 & 1.3 \\
\hline Tampa Bay & 13.4 & 0 & 13.2 & 9.5 & 5.9 & 1.9 & 1.6 & 0 & 3.8 & 0 & 2.3 & 3.8 & 10.4 & 5.5 & 25.3 & 3.3 \\
\hline Trinity & 5.1 & 0 & 6.5 & 5.5 & 3.2 & 1.5 & .4 & 7.5 & 6.2 & 3.5 & 3.9 & 21.7 & 19.6 & 6.2 & 7.9 & 1.3 \\
\hline Umpqua & .5 & 0 & 2.2 & .4 & .2 & .1 & .6 & .5 & 66.8 & 5.8 & 12.3 & 4.6 & 5.1 & .3 & .6 & .2 \\
\hline Waccamaw & 1.3 & 0 & 4.8 & 1.7 & .4 & .1 & .1 & 1.2 & 21.2 & 1.8 & 3.4 & 11.7 & .8 & 17.7 & 33.0 & .9 \\
\hline Withlacoochee & 1.4 & 0 & 9.8 & 2.6 & .8 & .1 & .3 & .1 & 19.6 & 0 & 3.0 & 3.0 & 21.6 & 1.7 & 30.7 & 5.2 \\
\hline Yellow & 2.3 & 0 & 6.3 & .9 & .2 & .1 & .3 & 2.4 & 44.5 & 4.9 & 10.0 & 3.9 & 7.3 & 6.6 & 9.7 & .6 \\
\hline Yellowstone & .5 & 0 & .6 & .2 & .1 & 0 & 1.0 & .2 & 12.6 & 0 & 34.5 & 42.2 & 1.6 & 4.5 & 1.3 & .6 \\
\hline
\end{tabular}


Table 5. National Land Cover Data classification schemes, 2001 (from U.S. Geological Survey, 2007).

\begin{tabular}{|c|c|}
\hline & Classification \\
\hline 11 & Open Water \\
\hline 12 & Perennial Ice / Snow \\
\hline 21 & Developed, Open Space \\
\hline 22 & Developed, Low Intensity \\
\hline 23 & Developed, Medium Intensity \\
\hline 24 & Developed, High Intensity \\
\hline 31 & Barren Land \\
\hline 32 & Unconsolidated Shore \\
\hline 41 & Deciduous Forest \\
\hline 42 & Evergreen Forest \\
\hline 43 & Mixed Forest \\
\hline 51 & Dwarf Shrub \\
\hline 52 & Scrub/Shrub \\
\hline 71 & Grasslands/Herbaceous \\
\hline 72 & Sedge Herbaceous \\
\hline 73 & Lichens \\
\hline 74 & Moss \\
\hline 81 & Pasture/Hay \\
\hline 82 & Cultivated Crops \\
\hline 90 & Woody Wetlands \\
\hline 95 & Emergent Herbaceous Wetlands \\
\hline
\end{tabular}

\section{Watershed Layers (Google Earth)}

In Google Earth, Watershed Layers contains folders with integrated characteristics similar to a GIS (including transparency). The Watershed Layers folders include watershed boundary, elevation, land cover, and EDNA synthetic channels (streams). The Boundary is a polygon generated by the watershed tool by using the EDNA database and represents the watershed basin in that it follows the drainage divide. The Elevation folder provides topographic information and is derived from the EDNA database. The Land Cover folder contains 1992 and 2001 land cover distributions. The Streams folder contains mean annual streamflow in cubic meters per second for the EDNA synthetic streams. Some of the channels in larger watersheds are generalized because of software limitation of vertices. The Population folder contains 1990 and 2000 average population densities. The Watershed Layers folders that contain temporal data, such as Population, can be independently toggled to determine change within the watershed (fig. 8).

\section{Watershed Characteristics (Google Earth)}

In Google Earth, Watershed Characteristics is a gateway to the elevation-derived watershed summary statistics. Selection of the link (either under the Temporary Places folder or the placemark in the center of the display window) accesses a Web link where the characteristics are available for analysis (fig. 9).

\section{Results}

Elevation-derived watershed basins and characteristics for major rivers of the conterminous United States contain characteristics for 77 watersheds. We have chosen to discuss the results of the elevation-derived watershed characteristics for the Chesapeake Bay only.

Land cover (1992) characteristics summarized for the Chesapeake Bay watershed indicate that the elevation-derived watershed basin primarily consists of forest, cropland, and pasture (fig. 10). Located in the highly urbanized eastern part of the country, the Chesapeake Bay watershed is composed of 3.9 percent urban land cover. Transitional and barren land account for a small percentage of this watershed area. Land cover (2001) characteristics generated from NLCD (U.S. Geological Survey, 2007) indicate the watershed still primarily consists of forest, cropland, and pasture (fig. 11).

In 1990, the average population density for the Chesapeake Bay watershed was 86.3499 persons per square kilometer (fig. 12). In 2000, the average population density had increased to 95.0975 persons per square kilometer (fig. 13). This direct spatial and temporal comparison is valuable for determining population change within a watershed and for evaluating population effects on watersheds.

The drainage area for the Chesapeake Bay watershed is 174,590 square kilometers with the minimum elevation at sea level and a maximum elevation of 1,481.10 meters (fig. 14). The minimum slope for the Chesapeake Bay watershed is 0 degrees, and the maximum slope is 69.3009 (fig. 15).

\section{Discussion}

The EDNA watershed delineation tool provided an avenue to delineate 77 watersheds that constitute the EDNA Watershed Atlas. The watershed delineation tool, however, is designed to create a watershed from any point within the conterminous United States. Therefore, additional watersheds and their characteristics can be added to the EDNA Watershed Atlas.

The number of watershed characteristics was limited by time and budgetary constraints. Those characteristics included in the EDNA Watershed Atlas are land cover, population, drainage area, elevation, slope, and other ancillary data including dams. After the development of the EDNA Watershed 
Table 6. Population characteristics for elevation-derived watersheds, 1990 (persons per square kilometer; from Gridded Population of the World, Version 3, 2005).

\begin{tabular}{|c|c|c|c|c|c|c|c|}
\hline Watershed & Minimum & Maximum & Mean & Watershed & Minimum & Maximum & Mean \\
\hline Altamaha & 2.444 & $1,999.390$ & 50.3785 & Ohio & 0 & $3,816.750$ & 48.5199 \\
\hline Apalachicola & 1.165 & $1,999.390$ & 53.6451 & Pajaro & .540 & $1,252.090$ & 50.1417 \\
\hline Atchafalaya & 2.086 & 781.483 & 24.4898 & Pascagoula & 2.805 & $1,069.560$ & 19.6377 \\
\hline Biloxi & 6.484 & $1,608.420$ & 87.9239 & Peace & 3.082 & $1,023.800$ & 54.9626 \\
\hline Brazos & .150 & $1,571.540$ & 15.9799 & Pee Dee & 5.371 & $1,368.370$ & 47.4044 \\
\hline Calcasieu & 2.073 & 870.525 & 22.4073 & Penobscot & 0 & 320.941 & 7.4835 \\
\hline Caloosahatchee & 1.824 & $1,256.530$ & 71.6347 & Platte & .032 & $3,225.680$ & 14.7907 \\
\hline Cape Fear & 4.607 & $1,490.400$ & 60.3852 & Puget Sound & .087 & $9,272.470$ & 108.6190 \\
\hline Chehalis & .555 & 601.184 & 21.4743 & Rainy & .001 & 375.450 & 1.4961 \\
\hline Chowan & 3.259 & 356.272 & 15.2804 & Roanoke & 3.324 & $1,280.440$ & 34.9882 \\
\hline Colorado & 0 & $2,869.010$ & 7.9348 & Rogue & .553 & 759.116 & 16.2251 \\
\hline Colorado-Texas & .162 & $2,014.810$ & 12.4362 & Sabine & 1.231 & $1,323.530$ & 23.6667 \\
\hline Columbia & 0 & $2,943.600$ & 9.3127 & $\begin{array}{l}\text { Sacramento/ } \\
\text { San Joaquin }\end{array}$ & .227 & $10,889.100$ & 42.8452 \\
\hline Connecticut & .380 & $4,322.990$ & 71.0533 & Saint John & 0 & 276.292 & 5.3935 \\
\hline Delaware & 1.515 & $7,773.130$ & 220.7280 & Saint Johns & 1.318 & $1,846.690$ & 99.2333 \\
\hline Edisto & 3.116 & 595.132 & 23.7199 & Salinas & .454 & $2,187.560$ & 22.6844 \\
\hline Eel & .398 & 150.419 & 4.4740 & San Francisco & 0 & $10,889.100$ & 768.4640 \\
\hline Kennebec & .006 & $1,064.340$ & 16.9063 & Souris & .323 & 938.823 & 3.1468 \\
\hline Klamath & .112 & 685.914 & 2.6981 & Suwannee & .560 & 867.353 & 15.4862 \\
\hline Lake Champlain & 1.058 & $1,755.680$ & 28.7681 & Tampa Bay & 0 & $2,328.500$ & 334.3530 \\
\hline Merrimack & 3.596 & $3,942.030$ & 135.1700 & Trinity & 0 & $3,035.130$ & 122.9840 \\
\hline Mississippi & .025 & $18,495.500$ & 21.4810 & Umpqua & .553 & 241.142 & 7.2061 \\
\hline Missouri & 0 & $3,225.680$ & 7.6948 & Waccamaw & 5.385 & 851.236 & 33.0933 \\
\hline Mobile & 1.786 & $1,592.850$ & 31.7039 & Withlacoochee & 9.231 & 702.942 & 44.4995 \\
\hline Navidad & 2.971 & $1,166.390$ & 11.7183 & Yellow & 2.924 & 418.485 & 16.6305 \\
\hline Neuse & 6.691 & $1,420.200$ & 70.5708 & Yellowstone & .032 & $1,109.990$ & 1.7943 \\
\hline Nueces & .047 & $2,908.900$ & 24.8054 & & & & \\
\hline Ochlockonee & 1.165 & $1,359.490$ & 22.7346 & & & & \\
\hline Ogeechee & .232 & $2,021.140$ & 26.2869 & & & & \\
\hline
\end{tabular}




\section{Elevation-Derived Watershed Basins and Characteristics for Major Rivers of the Conterminous United States}

Table 7. Population characteristics for elevation-derived watersheds, 2000 (persons per square kilometer; from Gridded Population of the World, Version 3, 2005).

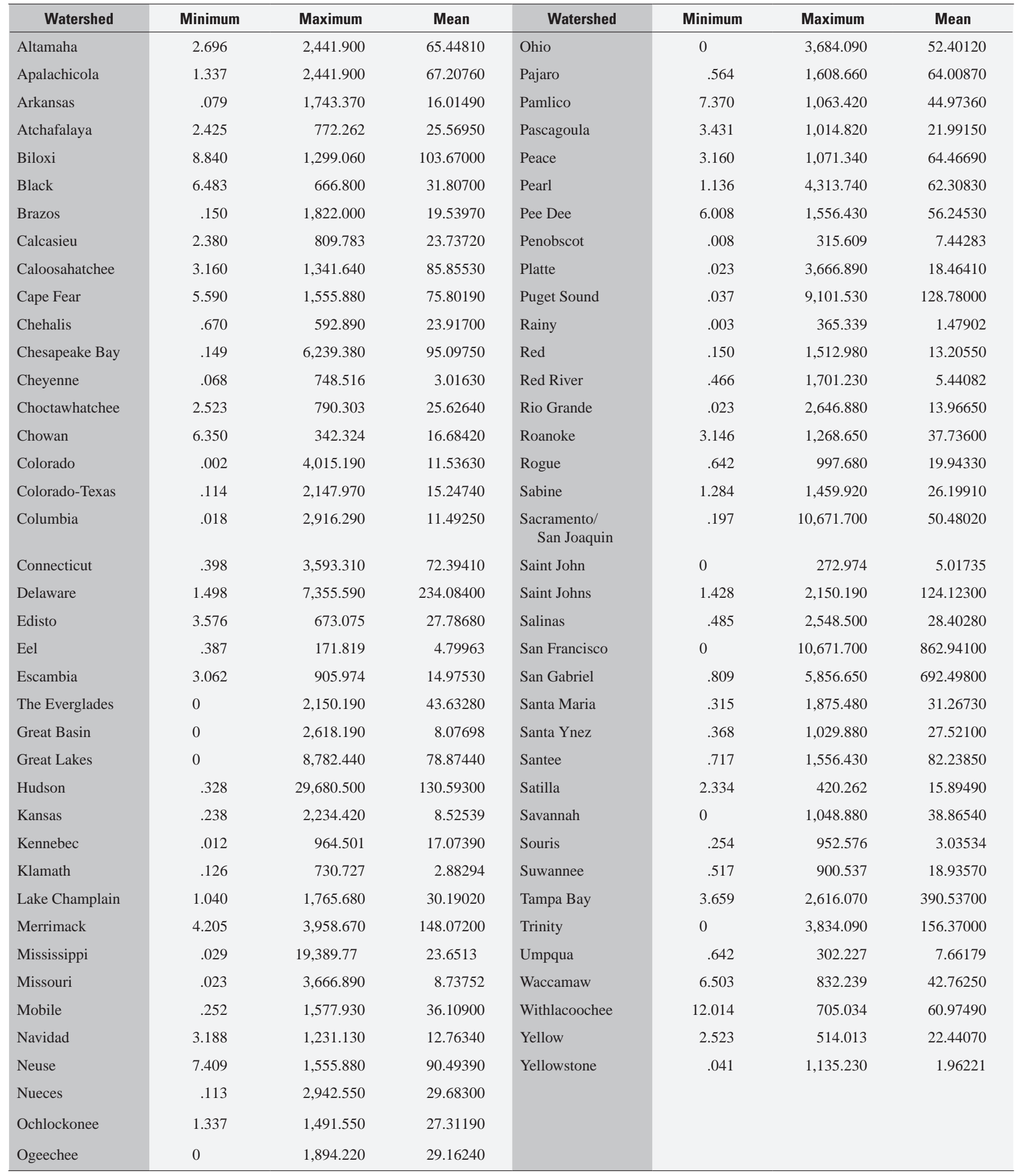


Table 8. Elevation characteristics for elevation-derived watersheds (EDNA).

\begin{tabular}{|c|c|c|c|c|c|c|c|}
\hline Watershed & $\begin{array}{c}\text { Area } \\
\text { (square } \\
\text { kilometers) }\end{array}$ & $\begin{array}{c}\text { Minimum } \\
\text { (meters) }\end{array}$ & $\begin{array}{c}\text { Maximum } \\
\text { (meters) }\end{array}$ & Watershed & $\begin{array}{c}\text { Area } \\
\text { (square } \\
\text { kilometers) }\end{array}$ & $\begin{array}{c}\text { Minimum } \\
\text { (meters) }\end{array}$ & $\begin{array}{c}\text { Maximum } \\
\text { (meters) }\end{array}$ \\
\hline Altamaha & $36,969.2$ & 0 & 508.12 & Ohio & $525,924.0$ & 86.99 & $2,035.83$ \\
\hline Apalachicola & $50,579.3$ & 0 & $1,348.87$ & Pajaro & $3,371.4$ & 0 & $1,597.36$ \\
\hline Arkansas & $405,274.0$ & 34.35 & $4,411.43$ & Pamlico & $8,908.9$ & 0 & 219.54 \\
\hline Atchafalaya & $14,369.3$ & 0 & 79.80 & Pascagoula & $24,562.6$ & 0 & 216.96 \\
\hline Black & $4,598.4$ & .05 & 131.96 & Pearl & $44,357.5$ & 0 & 220.06 \\
\hline Brazos & $11,917.4$ & 0 & $1,446.24$ & Pee Dee & $37,183.6$ & .53 & $1,255.77$ \\
\hline Calcasieu & $9,914.0$ & 0 & 142.75 & Penobscot & $21,641.5$ & 0 & $1,599.24$ \\
\hline Caloosahatchee & $4,695.2$ & -.35 & 48.74 & Platte & $220,529.0$ & 288.16 & $4,344.37$ \\
\hline Cheyenne & $65,489.3$ & 492.47 & $2,205.44$ & Red River & $104,877.0$ & 232.13 & 720.81 \\
\hline Choctawhatchee & $13,669.1$ & 0 & 210.88 & Rio Grande & $302,550.0$ & 0 & $4,361.49$ \\
\hline Chowan & $12,739.0$ & 0 & 214.21 & Roanoke & $25,216.4$ & 0 & $1,282.29$ \\
\hline Colorado & $625,052.0$ & 24.72 & $4,361.41$ & Rogue & $13,376.2$ & 0 & $2,887.44$ \\
\hline Colorado-Texas & $109,703.0$ & 0 & $1,384.99$ & Sabine & $55,122.6$ & 0 & 234.96 \\
\hline Columbia & $542,635.0$ & 0 & $4,391.03$ & $\begin{array}{l}\text { Sacramento/ } \\
\text { San Joaquin }\end{array}$ & $158,164.0$ & 0 & $4,410.10$ \\
\hline Connecticut & $27,714.9$ & 0 & $1,911.87$ & Saint John & $37,349.5$ & 31.86 & 852.84 \\
\hline Delaware & $35,504.2$ & 0 & $1,265.87$ & Saint Johns & $24,569.9$ & 0 & 94.50 \\
\hline Hudson & $34,920.5$ & 0 & $1,622.57$ & Satilla & $8,988.3$ & 0 & 112.57 \\
\hline Kansas & $155,489.0$ & 220.27 & $1,817.78$ & Savannah & $27,852.0$ & 0 & $1,672.74$ \\
\hline Kennebec & $25,492.5$ & 0 & $1,912.33$ & Souris & $43,557.2$ & 430.20 & 821.00 \\
\hline Klamath & $41,135.9$ & 0 & $4,301.61$ & Suwannee & $25,549.0$ & 0 & 158.74 \\
\hline Lake Champlain & $21,325.4$ & 28.87 & $1,623.40$ & Tampa Bay & $5,149.0$ & 0 & 80.20 \\
\hline Merrimack & $12,845.4$ & 0 & $1,597.49$ & Trinity & $60,672.3$ & 0 & 460.78 \\
\hline Mississippi & $3,171,588.0$ & 0 & $4,411.43$ & Umpqua & $12,104.7$ & 0 & $2,786.40$ \\
\hline Missouri & $1,325,810.0$ & 119.06 & $4,344.37$ & Waccamaw & $4,186.0$ & .53 & 41.46 \\
\hline Mobile & $113,316.0$ & 0 & $1,280.37$ & Withlacoochee & $5,384.3$ & 0 & 91.49 \\
\hline Navidad & $10,897.7$ & 0 & 180.70 & Yellow & $6,126.9$ & 0 & 160.15 \\
\hline Neuse & $14,335.9$ & 0 & 268.99 & Yellowstone & $181,388.0$ & 564.45 & $4,198.78$ \\
\hline Nueces & $91,967.9$ & 0 & 740.12 & & & & \\
\hline Ochlockonee & $6,773.5$ & 0 & 137.84 & & & & \\
\hline Ogeechee & $12,118.1$ & 0 & 225.66 & & & & \\
\hline
\end{tabular}


Table 9. Slope characteristics, in degrees, for elevation-derived watersheds (EDNA).

\begin{tabular}{|c|c|c|c|c|c|c|c|}
\hline Watershed & Minimum & Maximum & Mean & Watershed & Minimum & Maximum & Mean \\
\hline Altamaha & 0 & 56.723 & 2.4034 & Ohio & 0 & 85.539 & 6.9332 \\
\hline Apalachicola & 0 & 45.048 & 2.5798 & Pajaro & 0 & 54.621 & 12.8251 \\
\hline Atchafalaya & 0 & 16.073 & .3291 & Pascagoula & 0 & 30.056 & 2.4426 \\
\hline Biloxi & 0 & 12.781 & 1.4203 & Peace & 0 & 13.165 & .3133 \\
\hline Brazos & 0 & 39.087 & 1.5709 & Pee Dee & 0 & 51.816 & 2.9126 \\
\hline Calcasieu & 0 & 12.533 & .7617 & Penobscot & 0 & 67.428 & 3.4281 \\
\hline Caloosahatchee & 0 & 5.738 & .0781 & Platte & 0 & 78.849 & 3.8316 \\
\hline Cape Fear & 0 & 39.303 & 1.6845 & Puget Sound & 0 & 84.655 & 13.1218 \\
\hline Chehalis & 0 & 65.953 & 10.3737 & Rainy & 0 & 46.885 & 1.3063 \\
\hline Chowan & 0 & 21.775 & 1.4813 & Roanoke & 0 & 55.101 & 4.6593 \\
\hline Colorado & 0 & 88.926 & 8.3710 & Rogue & 0 & 68.504 & 16.7673 \\
\hline Colorado-Texas & 0 & 48.181 & 1.6211 & Sabine & 0 & 28.208 & 1.6643 \\
\hline Columbia & 0 & 85.397 & 11.6346 & $\begin{array}{l}\text { Sacramento/ } \\
\text { San Joaquin }\end{array}$ & 0 & 84.760 & 9.4769 \\
\hline Connecticut & 0 & 65.914 & 7.2958 & Saint John & 0 & 76.402 & 3.4405 \\
\hline Delaware & 0 & 66.791 & 4.4139 & Saint Johns & 0 & 20.819 & .4986 \\
\hline Edisto & 0 & 16.575 & .9905 & Salinas & 0 & 64.031 & 12.0949 \\
\hline Eel & 0 & 65.226 & 17.7757 & San Francisco & 0 & 53.465 & 9.9823 \\
\hline Kansas & 0 & 33.065 & 2.0485 & Savannah & 0 & 71.033 & 3.4455 \\
\hline Kennebec & 0 & 70.301 & 5.5723 & Souris & 0 & 49.881 & .6561 \\
\hline Klamath & 0 & 74.085 & 12.4504 & Suwannee & 0 & 19.328 & .6775 \\
\hline Lake Champlain & 0 & 67.814 & 6.7859 & Tampa Bay & 0 & 12.005 & .3874 \\
\hline Merrimack & 0 & 60.644 & 5.5055 & Trinity & 0 & 29.430 & 1.3535 \\
\hline Mississippi & 0 & 85.539 & 3.8619 & Umpqua & 0 & 68.545 & 18.0453 \\
\hline Missouri & 0 & 81.309 & 4.0302 & Waccamaw & 0 & 11.697 & .3336 \\
\hline Mobile & 0 & 54.426 & 4.0093 & Withlacoochee & 0 & 15.294 & .5734 \\
\hline Navidad & 0 & 12.464 & .5591 & Yellow & 0 & 18.128 & 1.8890 \\
\hline Neuse & 0 & 22.512 & 1.1622 & Yellowstone & 0 & 81.309 & 7.6615 \\
\hline Nueces & 0 & 58.237 & 1.8513 & & & & \\
\hline Ochlockonee & 0 & 24.734 & 1.5247 & & & & \\
\hline Ogeechee & 0 & 22.280 & 1.2536 & & & & \\
\hline
\end{tabular}


Table 10. National Inventory of Dams (NID) "Purposes" attribute categories.

\begin{tabular}{cl}
\hline $\begin{array}{c}\text { "Purposes" } \\
\text { Attribute }\end{array}$ & \multicolumn{1}{c}{ "Purposes" Attribute Categories } \\
\hline C & Flood Control and Storm Water Management \\
D & Debris Control \\
F & Fish and Wildlife Pond \\
H & Hydroelectric \\
I & Irrigation \\
N & Navigation \\
P & Fire Protection/Stock or Small Farm Pond \\
R & Recreation \\
S & Water Supply \\
T & Tailings \\
\hline
\end{tabular}

Atlas, the NLCD Retrofit Land Cover Change Data were released (http://www.mrlc.gov/multizone.php). Had these data been available during this research, they would have been included in the summarized statistics because they provide a more direct comparison with the 1992 NLCD (U.S. Geological Survey, 2007) categories.

\section{Conclusions}

Elevation-derived watersheds and characteristics for major rivers in the United States are developed to quantify EDNA hydrologic footprint characteristics so that researchers can evaluate the health of the Nation's watersheds by analyzing biological, physical, and anthropogenic change within watersheds. The watersheds are derived from the EDNA database by using the watershed generation tool to delineate watershed basins, or contributing areas, upstream from the mouth of major rivers of the conterminous United States. Watershed characteristics are selected to provide metrics for land cover distribution, population, drainage area, elevation, slope, and other ancillary data such as dams. The compilation of watershed basins and their characteristics are documented in an EDNA Watershed Atlas at http://edna.usgs.gov/ watersheds/.

The EDNA Watershed Atlas is a valuable resource for analyzing watershed characteristics and evaluating change within major river basins of the conterminous United States. This new database is broadly useful in landscape change modeling and subsequent monitoring activities in future years.

\section{References Cited}

Deichmann, U., Balk, D., and Yetman, G., 2001, Transforming population data for interdisciplinary usages from census to grid: The World Bank, 19 p. (Also available online at http:// sedac.ciesin.columbia.edu/gpw-v2/GPWdocumentation.pdf)

Franken, S.K., Tyler, D.J., and Verdin, K.L., 2001, Development of a national seamless database of topography and hydrologic derivatives, Proceedings of the 21st ESRI User's Conference, July 9-13, 2001, San Diego, CD-ROM. (Also available online at http://gis.esri.com/library/userconf/ proc01/professional/papers/pap730/p730.htm)

Gesch, D., Oimoen, M., Greenlee, S., Nelson, C., Steuck, M., and Tyler, D., 2002, The National Elevation Dataset: Photogrammetric Engineering and Remote Sensing, v. 68, no. 1, p. 5-33. (Also available online at http://www.asprs.org/ publications/pers/2002journal/january/highlight.html)

Gridded Population of the World, Version 3 (GPWv3), 2005, Gridded population of the world, version 3 (GPWv3) data collection: Center for International Earth Science Information Network (CIESIN), Centro Internacional de Agricultura Tropical (CIAT), July 2006, available online at http://sedac.ciesin.columbia.edu/gpw/index.jsp

Kost, J.R., and Kelly, G.G., 2001, Watershed delineation using the National Elevation Dataset and semiautomated techniques. Proceedings of the 21st ESRI User's Conference, July 9-13, 2001, San Diego, CD-ROM. (Also available online at http://gis.esri.com/library/userconf/proc01/ professional/papers/pap421/p421.htm)

U.S. Geological Survey, 2007, Multi-Resolution Land Characteristics (MRLC) Consortium, National Land Cover Database, available online at http://www.mrlc.gov

Verdin, K.L., and Greenlee, S.K., 2003, Continuous parameterization using EDNA, Proceedings of the 23rd ESRI User's Conference, July 7-11, 2003, San Diego, CD-ROM. (Also available online at http://gis.esri.com/library/ userconf/proc03/p0617.pdf) 


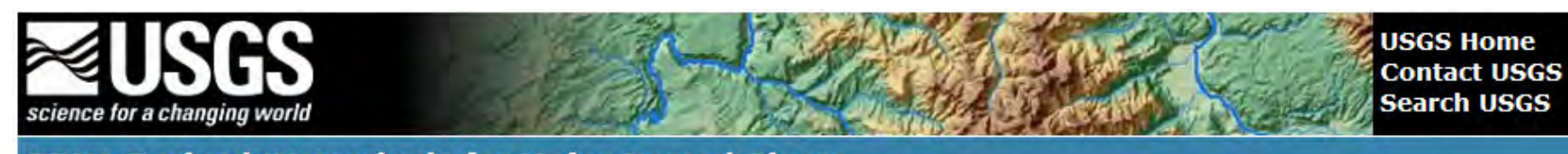

EDNA Derived Watersheds for Major Named Rivers

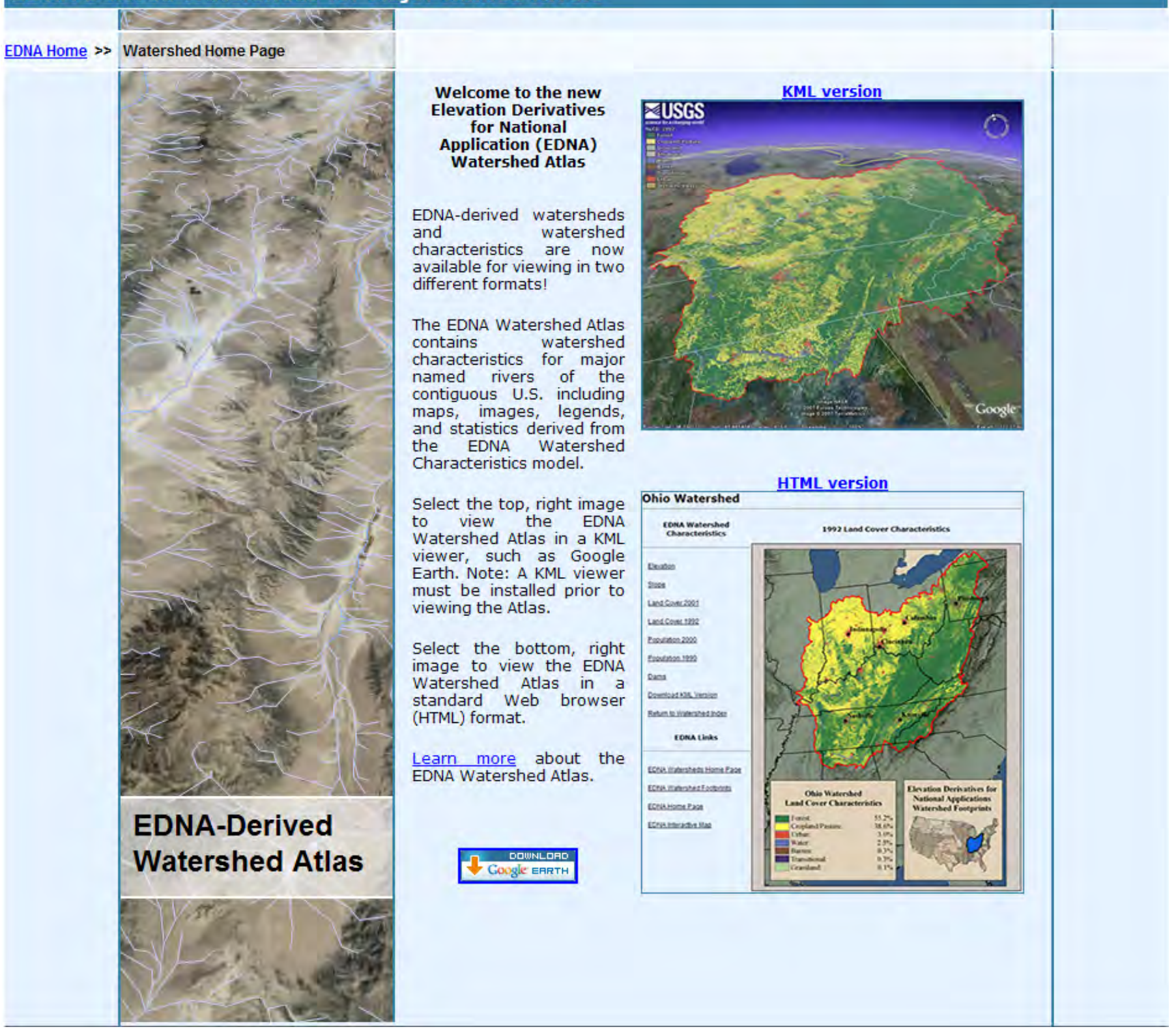

Figure 4. EDNA Watershed Atlas Web portal (http://edna.usgs.gov/watersheds) is accessible in a standard Web browser (HTML link) or with a three-dimension option, such as Google Earth (KML version link). 


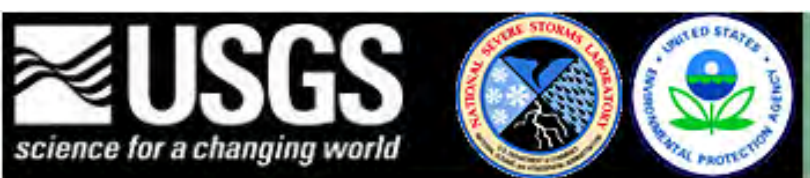

USGS Home

Contact USGS

Search USGS

\section{Elevation Derivatives for National Applications (EDNA)}

Home $\mid$ Backoround $\checkmark$ Data $\checkmark$ Applications $\checkmark$ Publications $\checkmark \mid$ Contact Us $\checkmark$

EDNA is a multi-layered database derived from a version of the National Elevation Dataset (NED), which has been hydrologically conditioned for improved hydrologic flow representation. The seamless EDNA database provides 30 meters resolution raster and vector data layers including:

- Aspect

- Contours

- Filled DEM

- Flow Accumulation

- Flow Direction

- Reach Catchment Seedpoints

- Reach Catchments

- Shaded Relief

- Sinks

- Slope

- Synthetic Streamlines

Hydrologically conditioned elevation data, systematically and consistently processed to create hydrologic derivatives,can be useful in many topologically based visualization and investigative applications. Drainage areas upstream or downstream from any location can be accurately traced facilitating flood analysis investigations, pollution studies, and hydroelectric power generation projects.

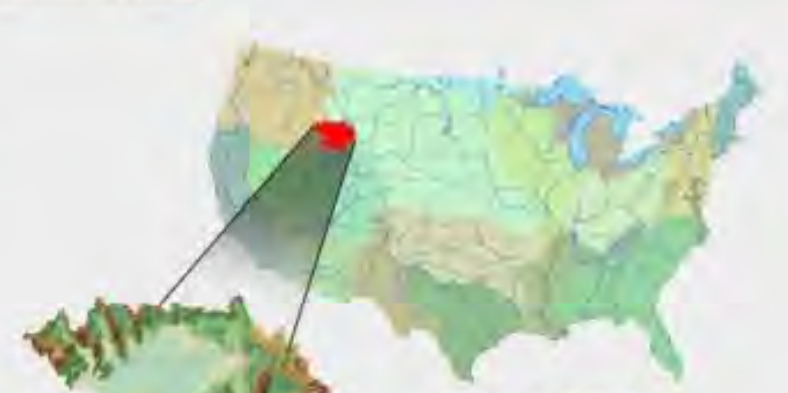

View EDNA Interactive Map

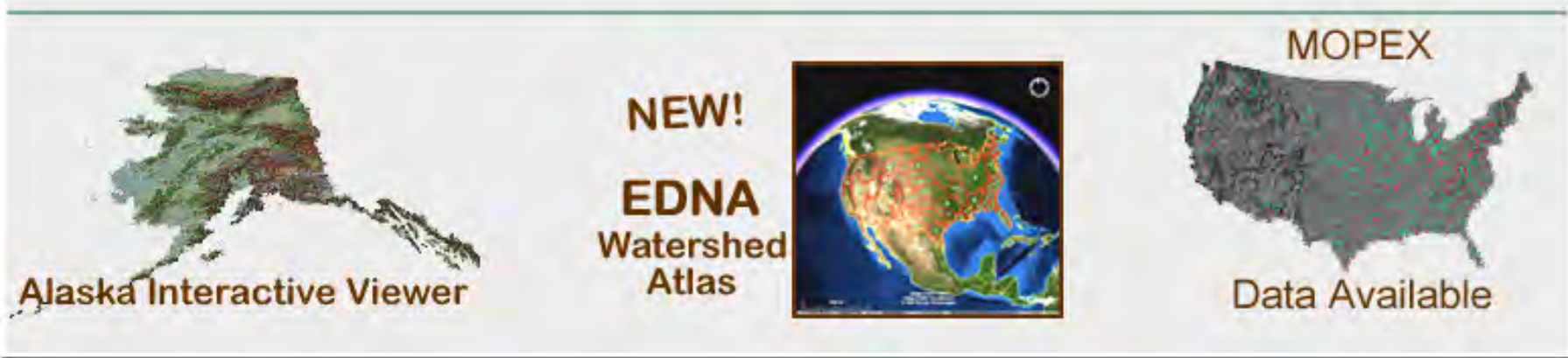

Figure 5. The EDNA Watershed Atlas is accessible from the EDNA Web site at http://edna.usgs.gov. 


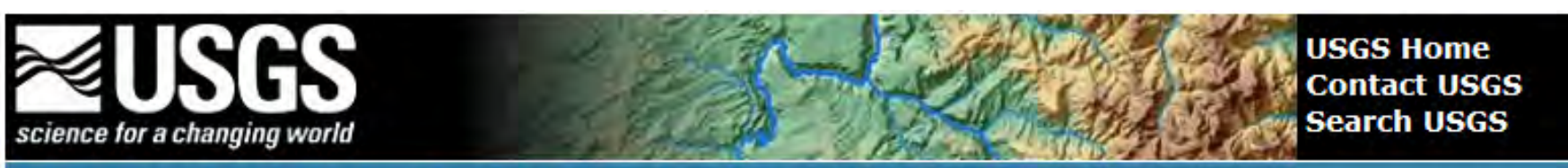

\section{EDNA Derived Watersheds for Major Named Rivers}

$\underline{E D N A \text { Home }} \gg$ Watershed Home Paqe $\gg$ HTML Watershed Index $\gg$ Chesapeake Bay Watershed Characteristics

\section{Chesapeake Bay Watershed}

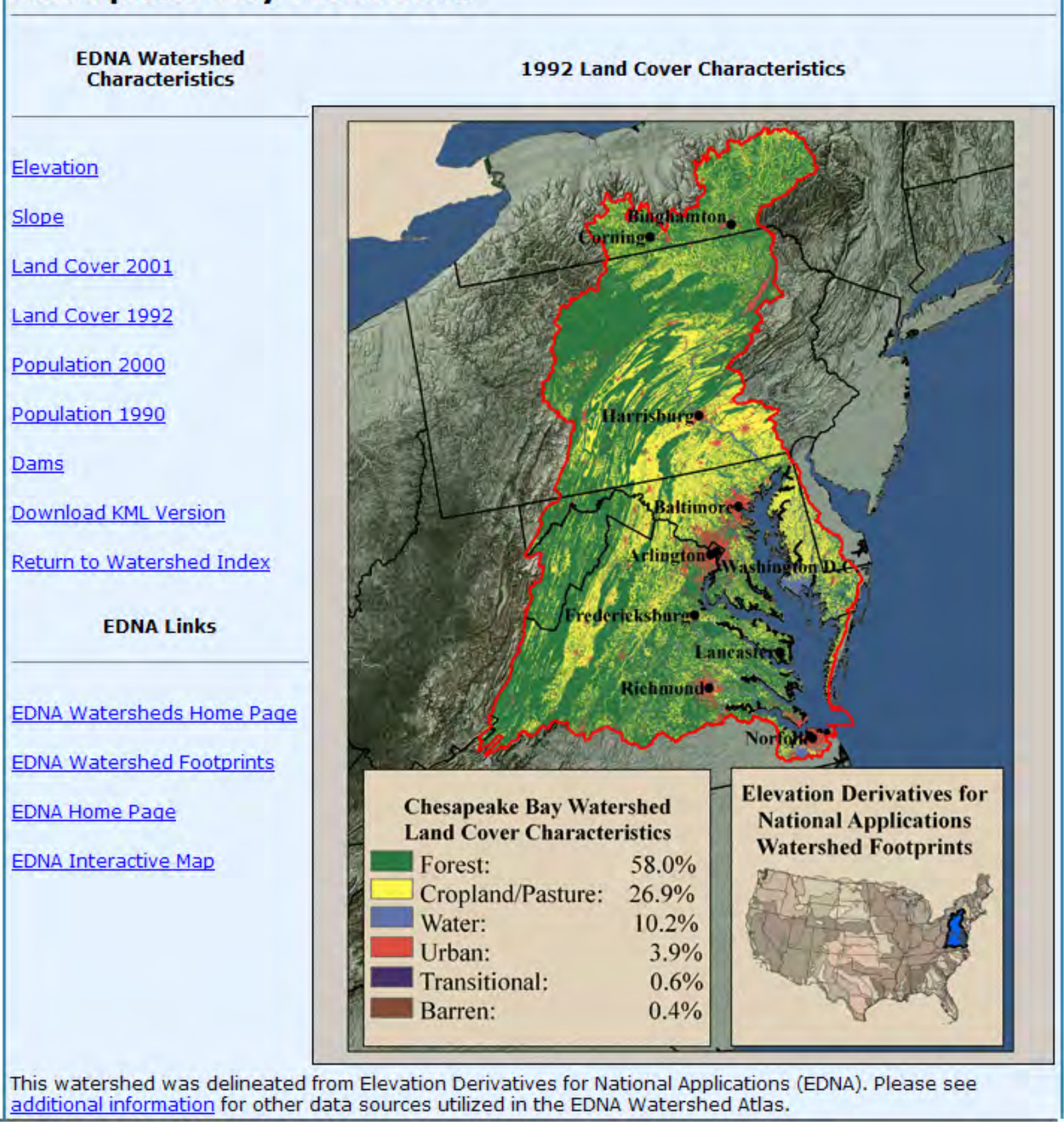

Figure 6. Land cover characteristics for the Chesapeake Bay watershed (U.S. Geological Survey, 2007). 


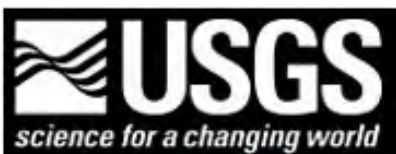

USGS Home

Contact USGS

science for a changing world

EDNA Derived Watersheds for Major Named Rivers

EDNA Home $\gg$ Watershed Home Paqe $\gg$ HTML Watershed Index $\gg$ Chesapeake Bay Watershed Characteristics

\section{Chesapeake Bay Watershed}

EDNA Watershed

Characteristics

Slope Characteristics

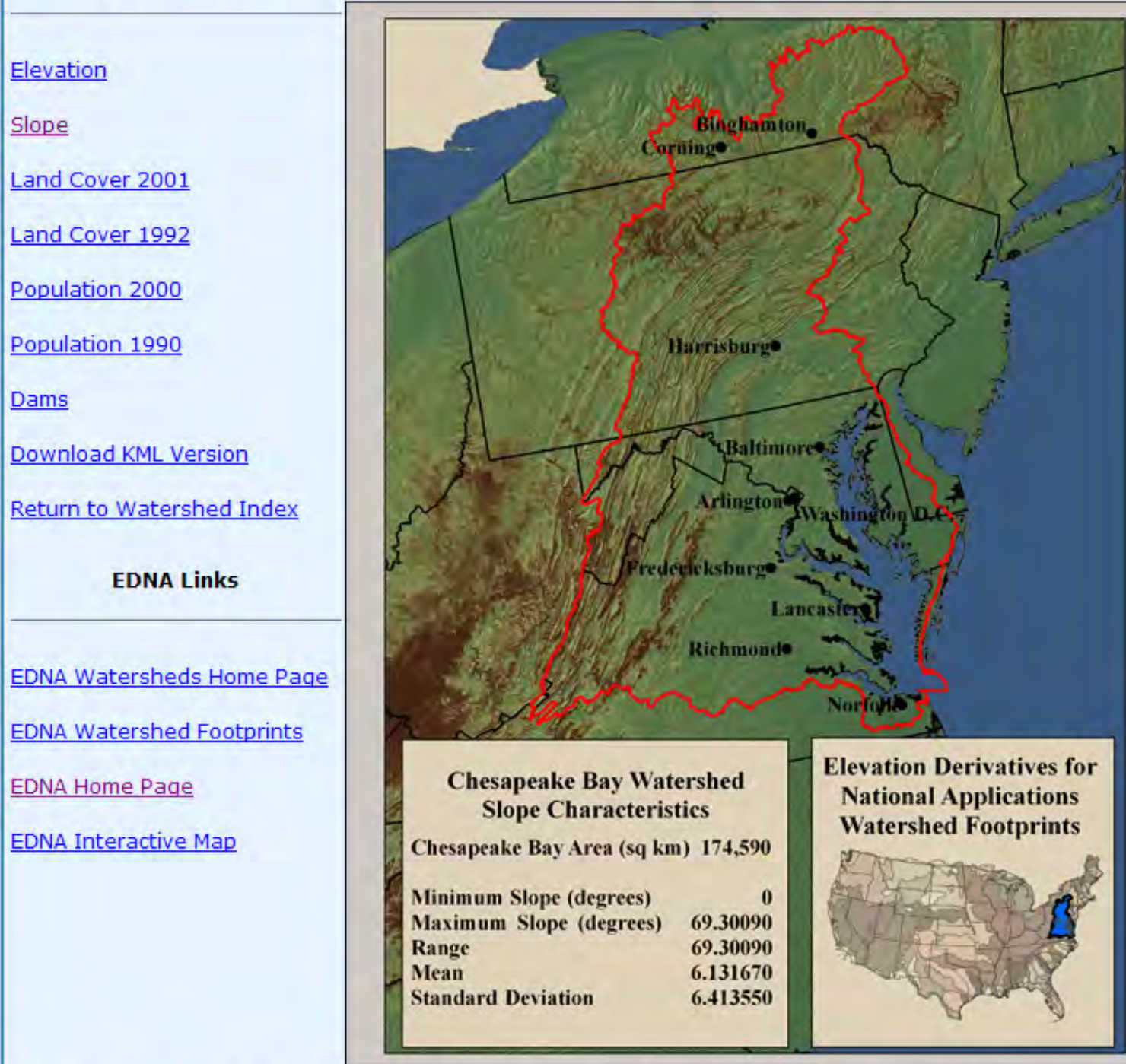

This watershed was delineated from Elevation Derivatives for National Applications (EDNA). Please see additional information for other data sources utilized in the EDNA Watershed Atlas.

Figure 7. Slope characteristics for the Chesapeake Bay watershed (EDNA). 


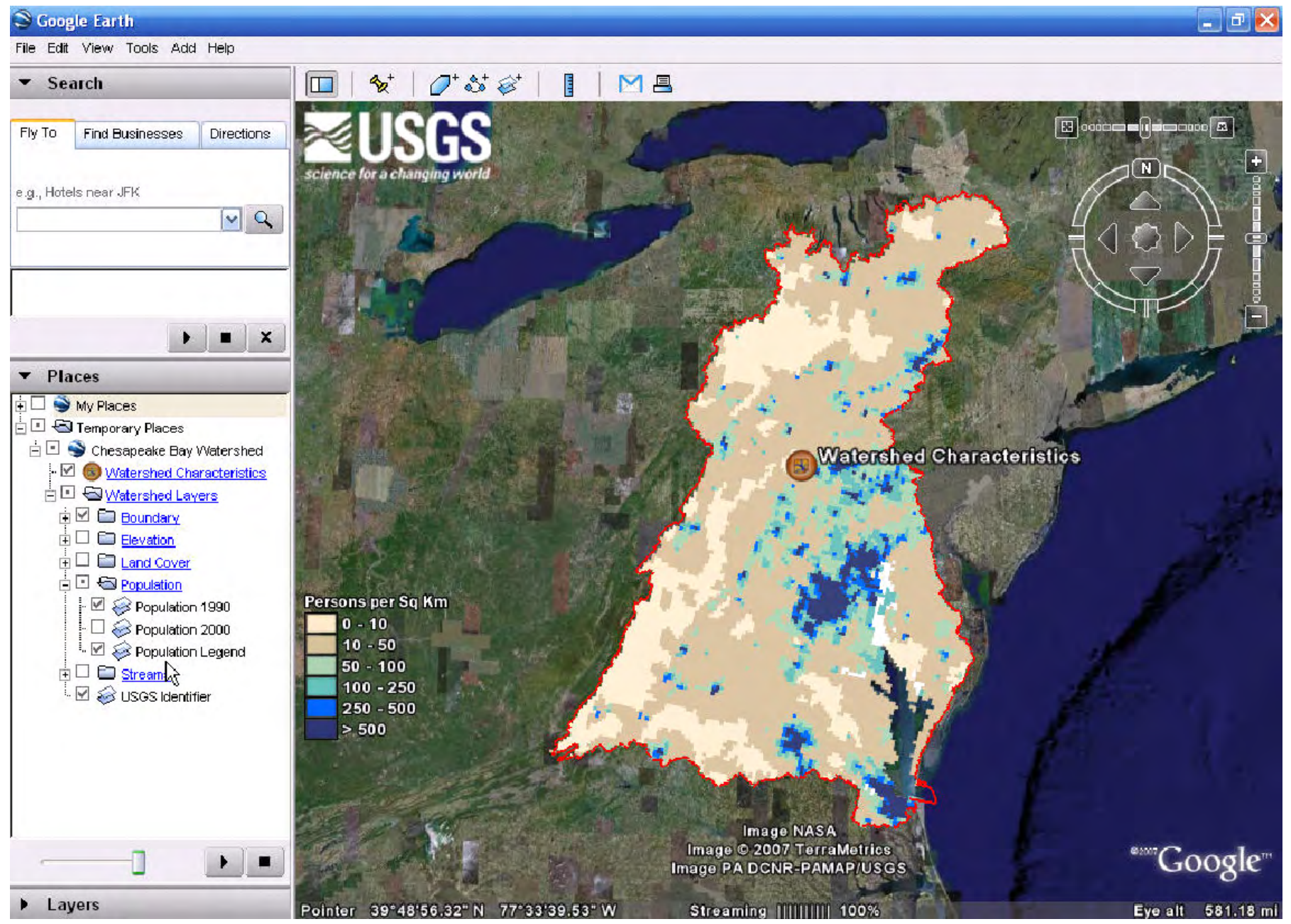

Figure 8. Population characteristics for the Chesapeake Bay watershed (Gridded Population of the World, Version 3, 2005). 


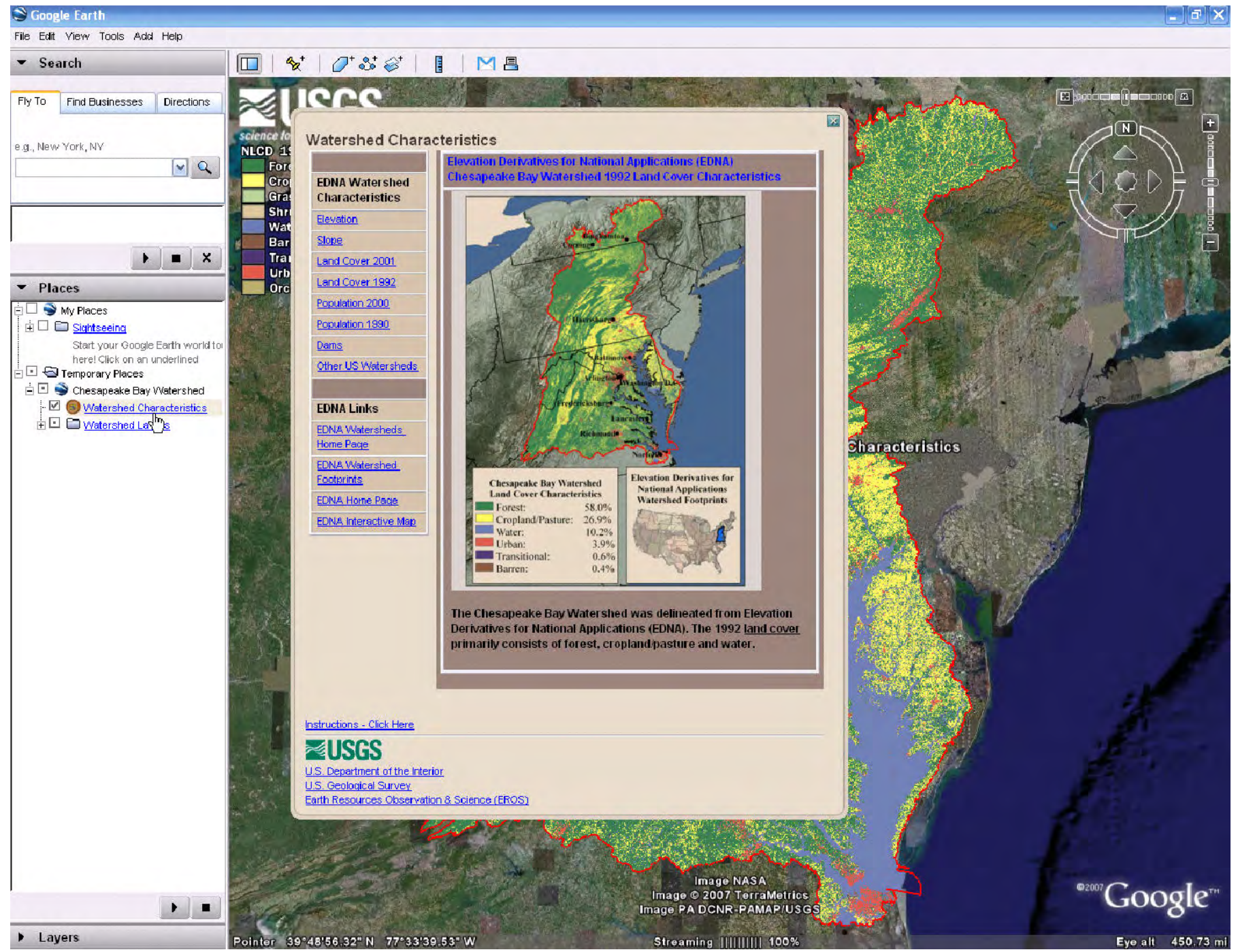

Figure 9. Characteristics for the Chesapeake Bay watershed accessible in Google Earth. 


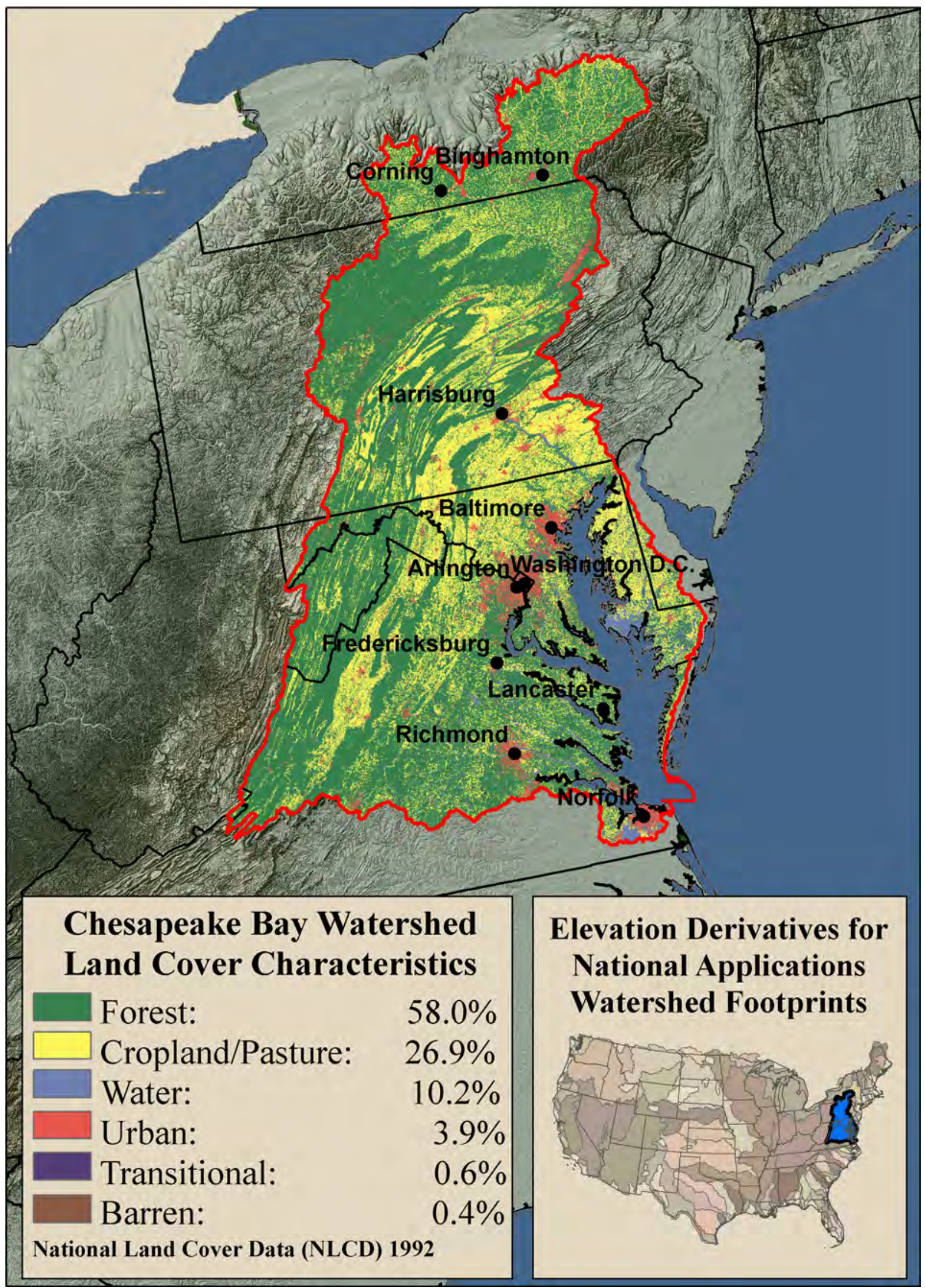

Figure 10. Land cover characteristics for the Chesapeake Bay watershed, 1992 (U.S. Geological Survey, 2007). 


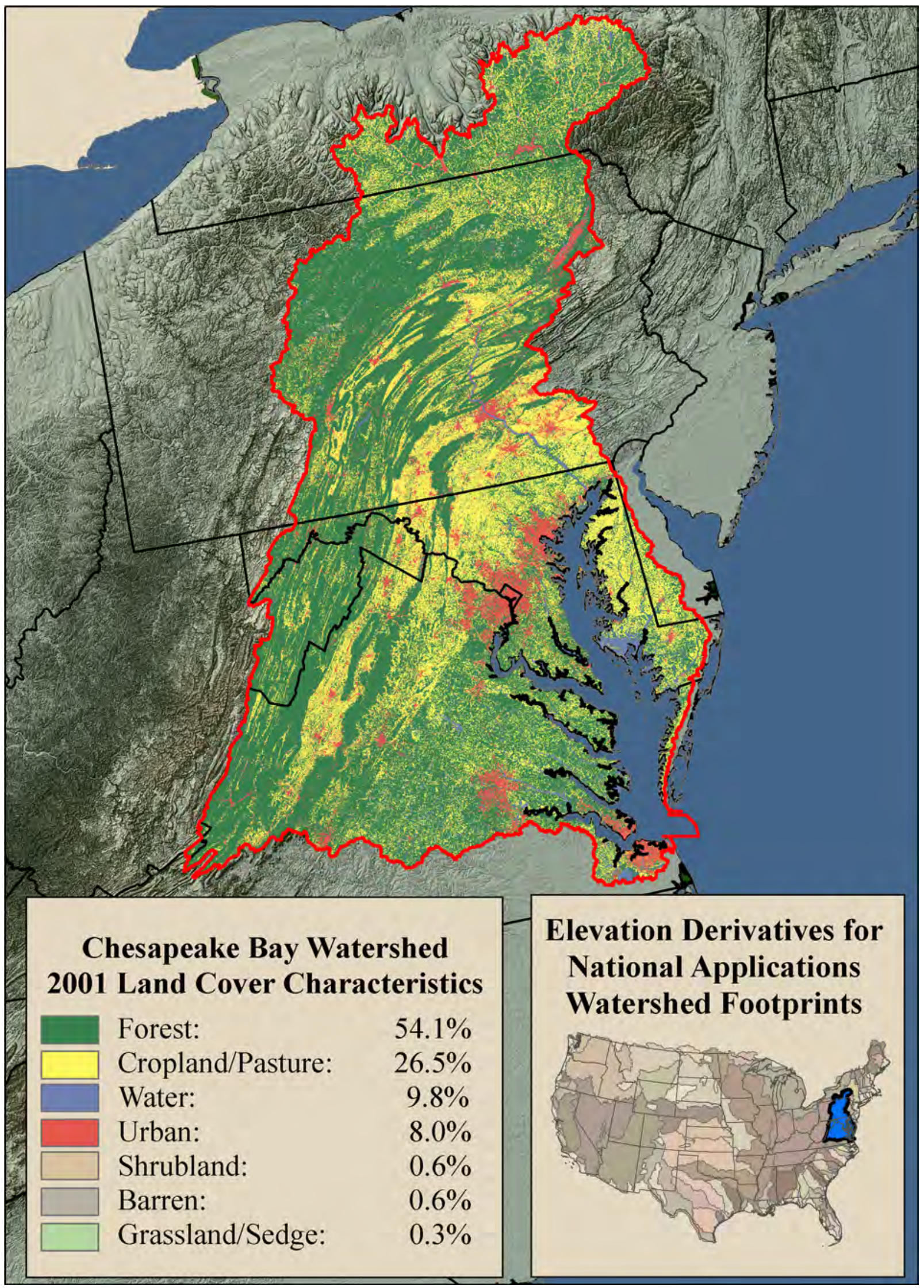

Figure 11. Land cover characteristics for the Chesapeake Bay watershed, 2001 (U.S. Geological Survey, 2007). 


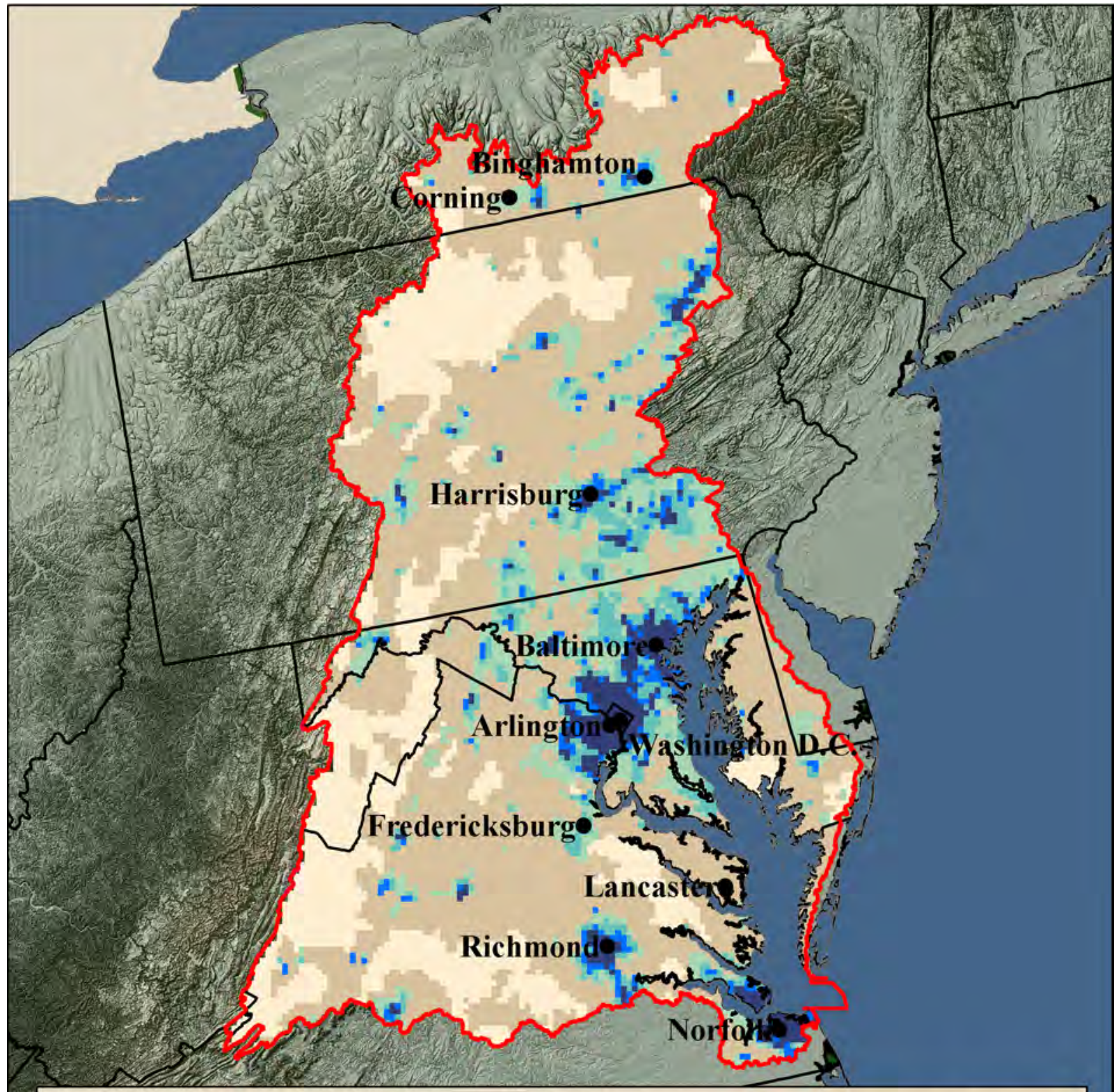

\section{Chesapeake Bay Watershed 1990 Average Population Density}

\begin{tabular}{|l|}
\hline $\begin{array}{l}\text { Persons (pr sq } \mathrm{km}) \\
86.3499\end{array}$ \\
$\begin{array}{l}\text { Area (sq km) } \\
\text { Minimum }\end{array}$ \\
$\begin{array}{l}\text { Maximum } \\
\text { Range }\end{array}$ \\
$\begin{array}{l}\text { Standard Deviation } \\
\text { 2816.590 }\end{array}$ \\
$\begin{array}{l}\text { Center for International Earth } \\
\text { Science Information Network } \\
\text { (CIESIN) }\end{array}$ \\
\hline
\end{tabular}

\begin{tabular}{|c|c|}
\hline \multicolumn{2}{|c|}{$\begin{array}{l}\text { Population Density } \\
\text { Persons Per Sq Km }\end{array}$} \\
\hline & $0-10$ \\
\hline & $10-50$ \\
\hline & $50-100$ \\
\hline & $100-250$ \\
\hline & $250-500$ \\
\hline & $>\mathbf{5 0 0}$ \\
\hline
\end{tabular}

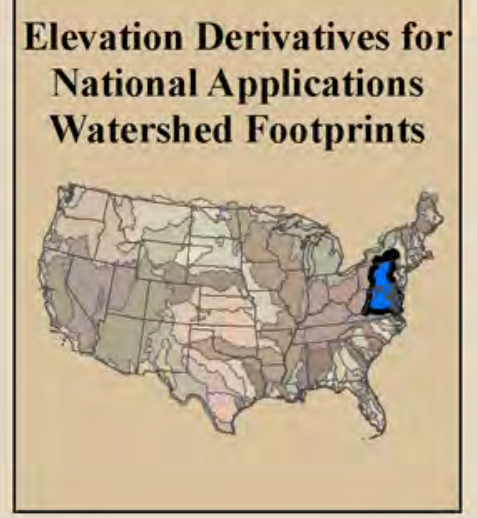

Figure 12. Population characteristics for the Chesapeake Bay watershed, 1990 (Gridded Population of the World, Version 3, 2005). 


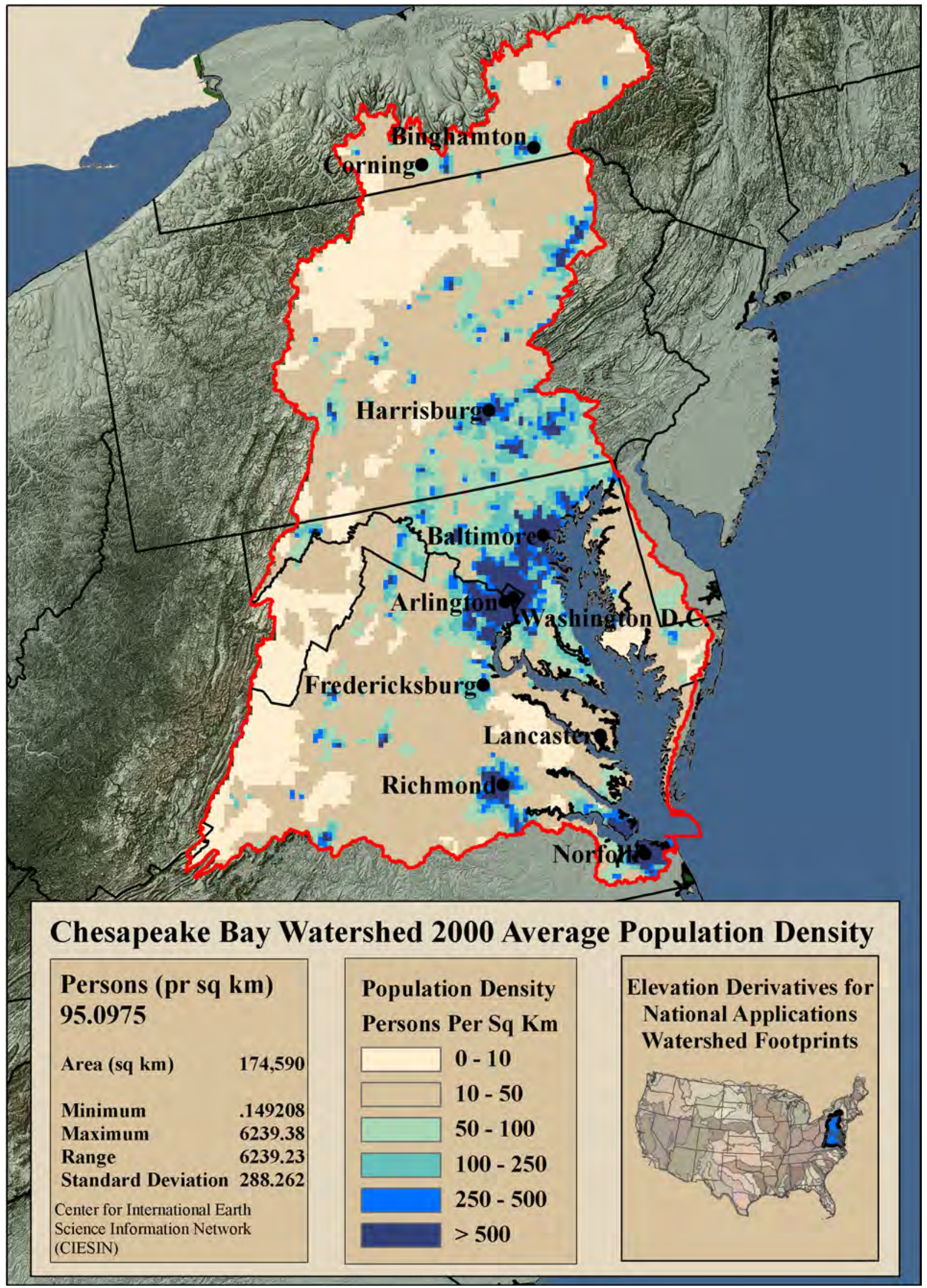

Figure 13. Population characteristics for the Chesapeake Bay watershed, 2000 (Gridded Population of the World, Version 3, 2005). 


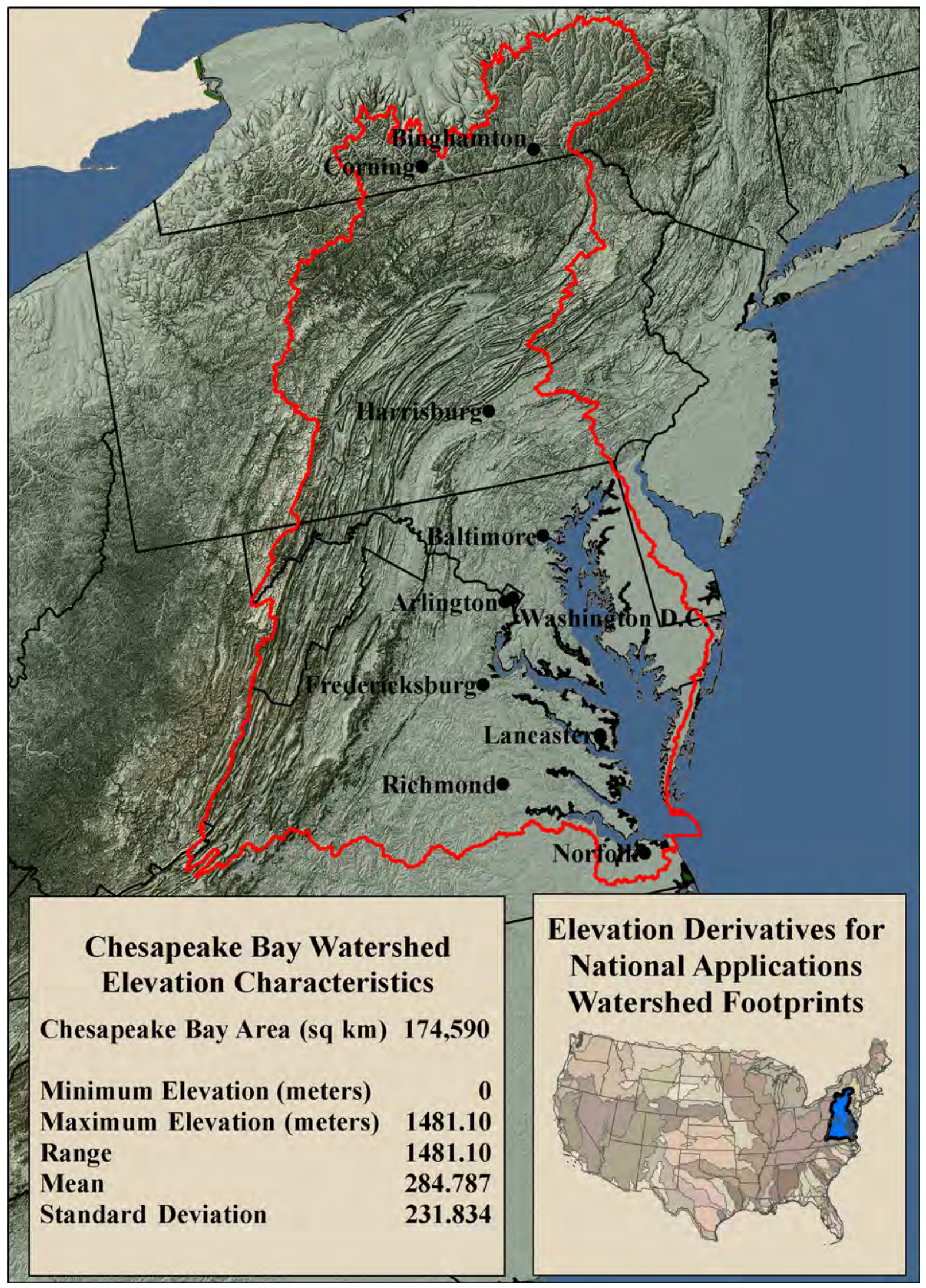

Figure 14. Elevation characteristics for the Chesapeake Bay watershed (EDNA). 


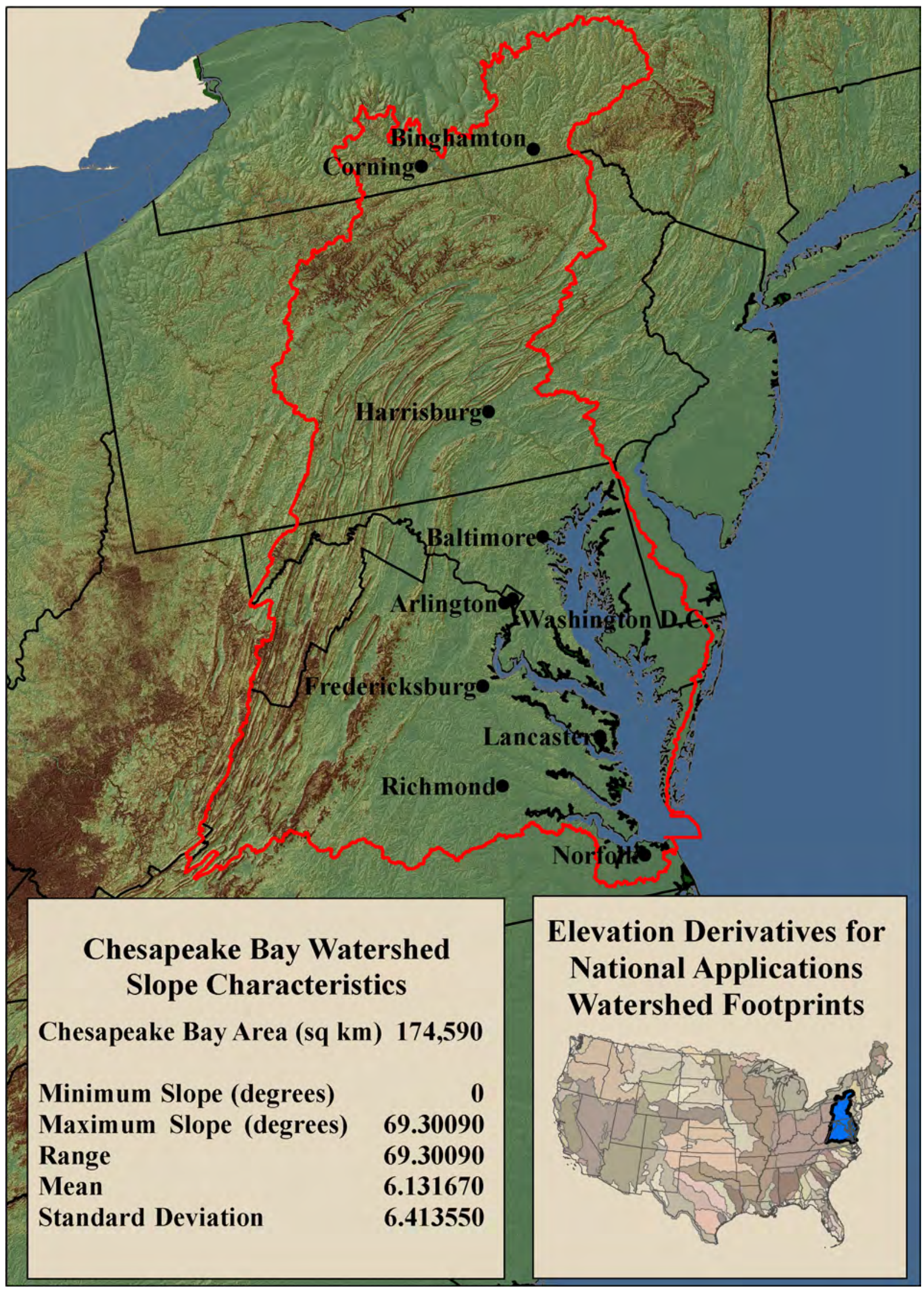

Figure 15. Slope characteristics for the Chesapeake Bay watershed (EDNA). 

Publishing support provided by:

Helena Publishing Service Center

For more information concerning this publication, contact:

U.S. Geological Survey

Center for Earth Resources Observation and Science (EROS)

47914 252nd Street

Sioux Falls, SD 57198-0001

(800) 252-4547

Or visit the Earth Resources Observation and Science Center Web site at: http://eros.usgs.gov/ 
